\title{
Back-off: Evaluation of Robot Motion Strategies to Facilitate Human-Robot Spatial Interaction
}

\author{
JAKOB REINHARDT, LORENZ PRASCH, and KLAUS BENGLER, \\ Technical University of Munich
}

\begin{abstract}
Standstill behavior by a robot is deemed to be ineffective and inefficient to convey a robot's intention to yield priority to another party in spatial interaction. Instead, robots could convey their intention and thus their next action via motion. We developed a back-off (BO) movement to communicate the intention of yielding priority to pedestrians at bottlenecks. To evaluate human sensory perception and subjective legibility, the $\mathrm{BO}$ is compared to three other motion strategies in a video study with 167 interviewees at the university and public spaces, where it excels regarding legibility. Implemented in a real encounter, objective motion behavior of 78 participants as a reaction to a stop-and-wait strategy, and two versions of BO (short and long), shows an improvement of the pedestrians' efficiency in the second encounter with the robot's short BO version compared to the stop strategy. Eventually, in the third encounter with all motion strategies, interaction causes only a small time consumption still required by the cognitive process of perceiving an object in the visual field. Hence, the design of kinematic parameters, $\mathrm{BO}$ path and time, exhibits the potential to increase the fluency of an interaction with robots at bottlenecks.
\end{abstract}

CCS Concepts: • Human-centered computing $\rightarrow$ Empirical studies in interaction design;

Additional Key Words and Phrases: Human-robot spatial interaction, legibility of motion, metrics for humanrobot interaction

\section{ACM Reference format:}

Jakob Reinhardt, Lorenz Prasch, and Klaus Bengler. 2021. Back-off: Evaluation of Robot Motion Strategies to Facilitate Human-Robot Spatial Interaction. ACM Trans. Hum.-Robot Interact. 10, 3, Article 23 (July 2021), 25 pages.

https://doi.org/10.1145/3418303

\section{INTRODUCTION AND RELEVANT WORK}

\subsection{Interaction with Service Robots}

Scientific discourse has postulated everyday human-robot interaction (HRI) in various situations for the past century, yet everyday interactions with robots are rather uncommon in the general population [2]. Nevertheless, researchers in academia and industry continue to develop economically feasible cases for the use of mobile service robots in daily life (e.g., parcels delivery $[42,45])$, accompanied by a considerable increase in the sales of mobile service robots [41]. However, the majority behind these sales are consumers who have willingly purchased a service robot for their homes, which are limited and controlled spaces. Apart from scientific studies and

Authors' address: J. Reinhardt, L. Prasch, and K. Bengler, Chair of Ergonomics, Technical University of Munich, Boltzmannstr. 15, Garching, Germany, 85748; emails: \{jakob.reinhardt, lorenz.prasch, bengler\}@tum.de.

Permission to make digital or hard copies of part or all of this work for personal or classroom use is granted without fee provided that copies are not made or distributed for profit or commercial advantage and that copies bear this notice and the full citation on the first page. Copyrights for third-party components of this work must be honored. For all other uses, contact the owner/author(s).

(c) 2021 Copyright held by the owner/author(s).

2573-9522/2021/07-ART23

https://doi.org/10.1145/3418303 

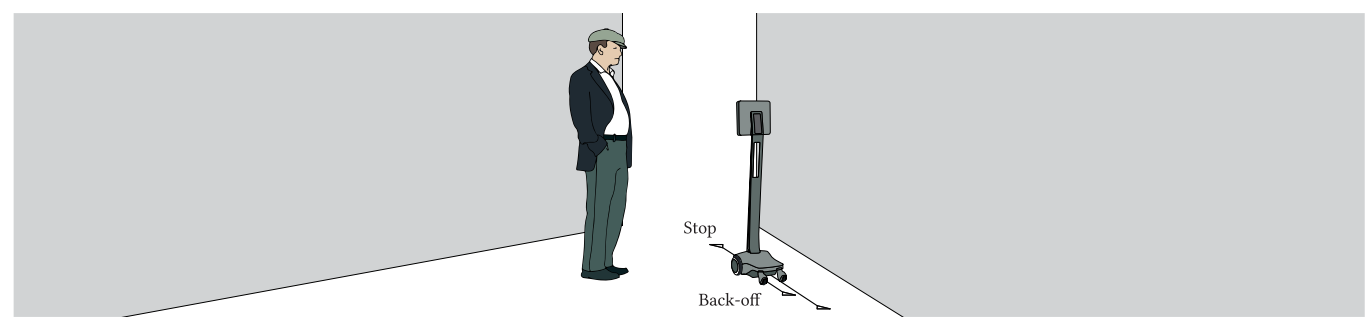

Fig. 1. Robots can show a back-off movement to communicate their intention of yielding priority to pedestrians at bottlenecks.

test programs, unplanned and unexpected interactions of pedestrian with service robots in pedestrian zones or public buildings are not yet commonplace, and this might be due to the complexity involved in interpersonal interactions between pedestrians and human road users. Managing bottlenecks [22] or solving deadlocks with other pedestrians poses everlasting difficulties for humans [12]. For fluent interactions, pedestrians rely on observing other pedestrians' motion to infer objectives, goals, and future trajectories $[25,27,56]$. Based on this, pedestrians plan their own trajectories with the goal to minimize "stop and go" or departure from their trajectory. Associated cooperation requires the agents in an encounter to manage interference to facilitate the individual activities or the common task [20]. In the case of crossing trajectories or at bottlenecks and doors, one agent has to partly recess from his or her planned trajectory and let the other agent move first [61]. To facilitate the order of passage, humans can use verbal communication, complementary gestures, or movements (e.g., a step backward) to yield priority to an interacting agent [56, 61]. In the same way, pedestrians must also cooperatively solve such spatial conflicts with robots (Figure 1); however, the individual level of previous experience with robots varies strongly from person to person $[42,53]$. Mobile robots exhibit a simplified embodiment compared to humans. Their movement capabilities are therefore more limited, which makes the use of complementary gestures neither applicable nor specific. Beyond this, verbal communication can be missed or perceived as inappropriate in crowded spaces. Thus, a novel motion language is necessary for fluent human-robot spatial interaction (HRSI) [45, 54]. For such a motion language to work properly, several prerequisites concerning traditional human-machine interaction have to be met. First and foremost, any kind of behavioral communication needs to be perceivable by the humans involved. Second, the intent behind a certain behavior should be comprehensible and enable both proper selection and execution of a corresponding response by the pedestrians.

\subsection{Information Processing}

Wickens et al. [63] divide human information processing into the consecutive steps of sensory perception, perception, response selection, and response execution, a model that provides a conceptual understanding and alignment of the processes involved in human-robot spatial interaction (HRSI). During the phase of sensory perception, the human eye plays the predominant role. For perception, the human derives knowledge from his or her long-term memory. Afterward, a feasible response is selected and then executed via movement. In the end, the process is updated by a constant feedback loop, which feeds the response by the environment back to sensory perception. This feedback loop continues while a pedestrian is interacting with a robot and is observing its movement. Here, similarities to anticipatory motion between humans are expected. Similar to the interaction with other humans, a person anticipates a robot's next movements and incorporates it into planning his or her own subsequent actions [21]. 


\subsection{Human Sensory Perception}

Human capabilities to see robotic motion pose requirements on the design of perceivable motion language. Generally, human perception can be classified into a visual field and a visual world. The human visual field roughly measures $180^{\circ}$ laterally and $150^{\circ}$ vertically. It possesses a central-toperipheral gradient of clarity. The visual field shifts when the eyes shift from one fixation point to another [14]. Foveal vision is characterized by low photosensitivity, high visual acuity, object identification and tracking, targeted peeking in a very small field of view, fixed perception of an object, and exact route guidance. To be able to see objects sharply, the entire eye must move and aim at the object. There are two types of quick eye movements: inspection saccades for exploring the environment and target saccades for targeted alignment with a distinct object [16]. Humans need at least $0.2 \mathrm{~s}$ to identify the area [62, 64]. In road traffic, a subsequent fixation period is usually in the range of 0.2 to $0.8 \mathrm{~s}$ [55]. This means that when an encounter with a robot is unplanned, it is expected to take approximately 0.4 to $1.0 \mathrm{~s}$ to scan the environment for an interfering robot and to fixate it. In contrast to quick eye movements, slow and sliding sequential moves are performed to track slow-moving objects [48]. Peripheral vision is characterized by high photosensitivity, low visual acuity, excellent motion detection, accidental detection in the large field of view, parallel perception of objects, and orientation in space. Hereby, above all, movement stimuli are perceived. Peripheral vision is fast but blurry. This is particularly true for directions of movement and velocity [15]. These two coexisting types of vision provide different qualities for the observer, and a combined mode of action is always present [65]. If the shifts between fixations are wide, the head also moves in the same direction as the eyes. In everyday life, it is rare for humans to perceive an environment with their heads motionless. Accordingly, head movements also have an effect on human perception. The visual field is deformed by these head movements. By tilting one's head to the side, the visual field becomes tilted sideways. The observer's visual world, however, is still upright [14].

In contrast to the visual field, the visual world has no boundaries. It is rather a conceptual model that includes an observer's previous knowledge and learned rules. It is trained by scanning the environment-that is, moving the eyes from point to point. The objects and surfaces are always clear and fully detailed in the visual world. If an object is not clear for the observer or only clear and detailed when fixated, the observer is in fact perceiving the object in the visual field, not the visual world [14]. One could assume that the human visual field defines an outer limit for the processing area. However, in the presented study we assume that the understanding of the scenario, the task, and the involved actors and thus the established visual world plays an important role. The latter can also be influenced beyond eye and head movements, particularly by attention processes and previously acquired knowledge. This may be the case when a pedestrian expects a robot to appear.

\subsection{Attention and Awareness}

An investigation of attention and awareness processes is a common prerequisite in the design of human-robot interaction (HRI) scenarios [7]. When designing motion for cooperation with pedestrians, it is useful to know what pedestrians are usually occupied with while walking. In fact, many studies share the finding that the pedestrians' attention is directed elsewhere (e.g., conversing with others, listening to an audio player, talking on a cell phone, or eating). Apparently, pedestrians pay little attention to walking [18]. Highly trained acts that have been repeatedly performed become skilled behavior that is executed without much cognitive workload [43]. Walking is such a skilled behavior, and thus it is performed automatically to a great extent. Accordingly, walking has been reported to require little to no attention [18]. Since a pedestrian's attention is unlikely to be directed at walking, the question remains whether pedestrians are fully aware of 
their surroundings, especially obstacles such as robots. Awareness is considered a prerequisite to understanding where we are, in which direction to move, and how to negotiate obstacles to safely arrive at a destination [10]. Attention may influence awareness, which exists in different gradations, ranging from no awareness at all to a fully conscious experience and perceptual description of a stimulus [50]. Additionally, humans can be partially aware [18, 28]. For example, a participant may express that a visual stimulus contains letters without being able to recall exact words [28]. Participants who act without being able to describe a stimulus may be considered as acting without awareness. Harms et al. [18] performed a study that focuses on whether pedestrians are sufficiently aware of their environment to avoid obstacles in a city, and whether secondary task engagement affects awareness. A $0.6 \times 1.09 \mathrm{~m}$ (width $\mathrm{x}$ height) obstacle signboard was placed on a pavement in a city center. Of all participants, $53.8 \%$ of 234 were not aware of the signboard while walking, yet nobody collided with it. Another $32.9 \%$ of participants were fully aware, and $13.2 \%$ were partially aware. Although the majority of participants are unaware of the obstacle, the lack of awareness does not imply any absence of cognitive processing for obstacle avoidance. Of all participants, $48.7 \%$ did not display any visible secondary tasks, and of those who engaged in such tasks, no effect on awareness or avoidance behavior was recorded. Most participants moved to avoid the obstacle between 1.5 and $0 \mathrm{~m}(40.6 \%)$ or 5 and $1.5 \mathrm{~m}(42.7 \%)$ in advance. The remaining participants adapted their trajectory between 10 and $5 \mathrm{~m}$ in advance. It seems that objects may guide a pedestrian's behavior without him or her being aware of the obstacle [18]. Hence, the degree to which participants are consciously aware of a robot in an unplanned encounter, despite having perceived it sensorily, may be debatable.

\subsection{Interpersonal Spatial Interaction}

In accordance with previous research, processes that are known to guide and steer human interpersonal interaction can be applied in HRSI [4, 54, 61]. Locomotion parameters and adaption strategies for collision avoidance have previously served as input for the design of convenient robot behavior [61]. Humans perceive movements via their underlying goals [56]. Besides understanding what another actor is doing at a certain moment, this includes being able to predict what he or she is going to do next. To support this, observed motion activates an existing mental model of the intended goal. In cooperative control strategies between humans, the intended goals of the other are more important for an observer's reaction than current events [27]. In unambiguous situations, mental models are already activated before the action is actually visible [56]. However, transferring this assumption directly to interaction with automated robots may be critical, since Sebanz and Knoblich [57] and Vassallo et al. [61] found that the actions humans anticipate from another actor differ, depending on whether they are interacting with another human who is perceived as acting intentionally or unintentionally or a machine with its artificial nature.

Human motion patterns are a highly trained behavior. Thus, the "what," "when," and "where" of human joint action of Sebanz and Knoblich [57] become increasingly important also in HRSI. When interacting with a robot, humans need to know what a robot intends to do. Humans need to know when a robot will perform an action, especially when timing an order of passage at a bottleneck. Furthermore, humans need to know where a robot will yield priority to them to know where possible trajectories to pass the robot lie. To evaluate the "what" and "when," research has previously focused on verbal communication as a modality to agree on a joint action [8]. However, Sebanz and Knoblich [57] believe that exclusive verbal exchange is not sufficient to achieve the attunement required for joint action. Body language and the study of motion is deemed to be faster than verbal communication [57]. When individuals have to time their actions in relation to those of others, language can be too slow to overcome a lack of shared internal processes [27]. It is thus not surprising that humans solve interpersonal spatial conflicts in public spaces, mainly by 


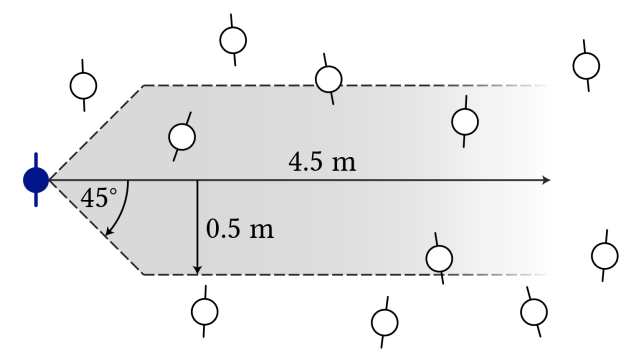

Fig. 2. The information process space (IPS) (grey) is a 4.5-m long and 1-m wide cone-shaped area with an opening angle of $45^{\circ}$ in front of a pedestrian (blue), where objects or other pedestrians (white) are perceived as potential obstacles when future trajectories are planned [26].

observing the others' motion [25]. Targeting "where" to guide interaction, the field of proxemics describes human preference, perception, and spatial utilization of interpersonal distances [47]. These are influenced by culture, relationship, activity, and emotion in everyday situations [17]. Hall et al. [17] describe relevant areas in the shape of concentric circles, whereas the personal space model of Tomari et al. [59] defines a personal space around humans as a combination of two ellipses that depend on head orientation and movement direction. Lam et al. [30] presented that the human-sensitive field is deformed by speed in an egg-shaped manner. Kitazawa and Fujiyama [26] propose an information process space (IPS) for pedestrian interpersonal interaction. It designates a space in which pedestrians perceive objects or other pedestrians as potential obstacles while planning future trajectories. Through several empirical studies, the authors model a coneshaped area with an opening angle of $45^{\circ}$, a $4.5-\mathrm{m}$ longitudinal length (in line with the findings of Harms et al. [18]), and a 0.5-m lateral width in front of the moving person (Figure 2).

\subsection{Human-Robot Spatial Interaction}

Robot behavior has to be designed taking into account the proximity and the intended or unintended interference of the two partners. In human-robot cooperation, human and robot share a (work)space at the same time and aim to achieve a common goal or task [52], so an interference is planned. As Hoc [20] put it, the common task should be facilitated by good management of the interference by the agents in an encounter, so successful cooperation can be accomplished. To achieve this, humans must understand the intent and trust the behavior of the robot [1]. The type of cooperative scenarios developers of robotic systems deal with is linked to human roles around robots. Roles have been categorized into supervisor, operator, mechanic, bystander, and peer [53]. Bystanders are humans whose planned paths interfere with those of a robot, and who are not informed about an encounter with the robot, such as humans in a pedestrian zone. The goal in such cases should not be to regulate human motion and behaviors, but to develop plausible rules for robots with the potential to maintain safe and smooth navigation for all users of public spaces [30]. Lam et al. [30] propose three such rules for robotic systems' motion strategies:

(1) Collision-free rule: A robot has to maintain safety and be able to reach the goal destination.

(2) Interference-free rule: A robot should not enter a person's personal space unless its task is to approach that person.

(3) Waiting rule: Once a robot enters a person's personal space, it has to stop and wait for a threshold time.

In encounters, an essential requirement for robot motion is to prevent collisions, instill confidence, and introduce minimum distress for humans [42], which corresponds to rule (1). Rule (2) 
may be impracticable in narrow spaces, and at bottlenecks, and rule (3) must be called into question for human-side interpretation. A simple pause and wait can lead to misunderstandings and decreasing acceptance, as information from the movement itself is missing. Especially in the case of short-term and time-critical adjustments, robot motion language for nonverbal communication must be designed in such a way that any specific intention becomes apparent [6, 9, 25, 42, 44, 45]. If an observer can infer a robot's intention efficiently and confidently from observing its unobstructed and undistorted motion, the movement is called legible [9, 33, 40]. Human biomechanics enable a large repertoire of social cues such as gaze direction, pointing gestures, and postural cues [56]. However, even simple objects in motion can be manipulated to communicate intentions through small movements [19].

Pacchierotti et al. [42] found concerns about the high degree of adaptivity achieved with a robotic system. Highly adaptive robots track people's actions in high frequency and adapt their own behavior afterward. A possible lack of transparency can lead to decoupling and distraction of the human partner, as the robot often changes its trajectory so that humans cannot follow in a precise manner [13]. Accordingly, predefined and recurring short-term movement cues (MCs) could be used by nonhumanoid robots with simple embodiment as nonverbal signaling method to convey an intention to pedestrians [7, 11, 44, 45]. The state-of-the-art applied human-aware navigation planning does not yet take into account the concepts of legibility and cues [29]. In laboratory settings, Faria et al. [11] have tested an movement cue (MC) to convince participants to follow a robot by moving toward and away from a person, and Kruse et al. [29] applied MCs in a human-robot path crossing scenario. Here, the robot showed minor accelerations during a longer-term planning algorithm. Both authors experienced weakened results by confounding the cues with other robot behavior, and thus a clear alignment of effect to the planned MC is not possible [11, 29]. Vassallo et al. [61] extended the human-robot path crossing scenario, focusing on the preferred order of passage depending on the robot's replication of the human's collision avoidance strategy. Limited by a very small sample size $(\mathrm{N}=10)$, they indicate an equal distribution of participants who decided to move in front of the robot and the ones who waited. The authors conclude that humans like to preserve their initial role in the encounter, which depends on the order of arrival at the crossing section.

Inspired by human hand hesitation movements [37], Reinhardt et al. [44] applied a back-off (BO) MC with an industrial robot's tool center point. The back-off (BO) is a backward movement along the original robot trajectory. It is executed to communicate the intention of yielding priority to the interacting human when a collision has to be avoided. In their study, the BO increased human subjective trust in the robot, compared with a stop-and-wait strategy [44]. In Sadigh et al. [49], a BO movement emerged from autonomous planning in a traffic scenario. Since pedestrians show similar adaption strategies in a crossing scenario [61], such BOs may also be used by mobile robots, and tested in the HRSI scenario of human and robot arranging an order of passage at a bottleneck.

So far, many studies in HRSI follow a rather static approach, where only one of the two interacting partners moves [38]. Here, social distances between robots and humans play a fundamental role [31]. Perceived robot performance is a predictor for preferred distances in the studies of Mead and Matarić [35]. Lauckner et al. [32] investigate a mobile robot as transport system in hospitals in a hallway-like setting. Here, the goal was to develop behavior that is safe and perceived as socially proper. They highlight the importance of motion and minimum acceptable frontal and lateral distances between human and robot. The static participants controlled the robot remotely, adjusting the stopping distance between them and an approaching robot. Additionally, the lateral passing distance between them and the passing robot was adjusted by the participants. Typical accepted frontal distances range from $M=0.73 \mathrm{~m}$ to $M=0.82 \mathrm{~m}$. Lateral distances range from 
$M=0.37 \mathrm{~m}$ to $M=0.41 \mathrm{~m}$. The differences in these parameters support the idea of the egg-shaped model of Lam et al. [30] or an elliptical model that Tomari et al. [59] proposed as human-sensitive fields. In another hallway-like setting, Pacchierotti et al. [42] tested the scenario of a human-robot passing scenario in a frontal encounter. Here, both human and robot were in motion. They found that accepted lateral passing distances range from 0.2 to $0.4 \mathrm{~m}$, where results are influenced by interpersonal preferences and perception. Most participants prefer wide evasion, although some criticize unnecessarily wide paths. Their robot behavior and results were also influenced by the corridor width. A preference for wider evasion values supports the validity of the IPS of Kitazawa and Fujiyama [26], where everything within a $0.5-\mathrm{m}$ lateral distance would be considered an obstacle in this context. Trinh et al. [60] target deadlocks that occur when a robot and a person enter a narrow passage in the same or opposing directions. They observed 157 persons as potentially causing deadlocks with the robot and 35 actually doing so. Their main contribution is an algorithmic formalism that classifies these two situations (opposing/same direction) for the robot control; however, despite it being considered necessary for future investigations, no findings on human abilities to recognize the robot's intention to either wait or to queue with the person were investigated [60].

Morales et al. [38] develop a human computation model for side-by-side walking with a robot. The human motion behavior was acquired by recording trajectories of humans walking in pairs. They implemented the model into robot control that can mimic human behavior when moving side by side in a corridor in a mall. For robot control and collision-free navigation, it is necessary to measure and understand human behavior [30]. Inaccuracy of human tracking leads to deficient performance in HRSI. Lam et al. [30] highlight that with more accurate human motion tracking, they might be able to derive human intentions from their movements and use this information for robot navigation.

\subsection{Goal of the Article}

As part of operation in public areas, robots will encounter bystanders who have no or limited prior exposure to robots $[42,53]$. It is important that their efficiency is not harmed, and utilizing robotic motion may be a convenient way to achieve this, that complies with human learned behavior [7, $25,61]$. Some authors provide reference values for the distances that robots should keep from humans in rather static HRSI scenarios [32, 42]; however, their experiments do not investigate human recognition of robot motion intentions and the corresponding human behavior [60]. Other studies investigate $\mathrm{MC}$ recognition but lack a study design that allows for conclusions on the effect of an MC exclusively $[11,29]$. Others monitor the order of passage, although small sample numbers are observed [61]. This gap should be closed by investigating human subjective and objective reactions to a single $\mathrm{MC}$ in front of a bottleneck where the arrangement of an order of passage is necessary and both human and robot are in motion. To prevent decoupling of the interacting humans due to overly adaptive robot behavior [13,42], the use of predefined movements for fluid interactions is applied. The rule for robots to simply stop and wait [30] to yield priority to humans is put into question, and in addition to earlier studies with an industrial robot [44], a robot's BO has the potential for legible communication at bottlenecks also in the domain of mobile robots. To conclude this, the requirements for legibility have to be met [9]. Motion has to be designed so that its underlying intention is inferred confidently and it leads to a higher efficiency, which is a common metric in HRI research [9, 11, 44, 58]. Therefore, the following two research questions are established:

(1) Can pedestrians infer the intention "yielding priority" from the BO movement?

(2) Does backing off lead to higher pedestrian efficiency compared to a stop-and-wait strategy? 
As knowledge is acquired about the robot during the experiment, a shift of perception from visual field to visual world is expected [14]. Furthermore, associated attention and awareness processes are considered in the study design to establish comparable surroundings for the participants while allowing them to be aware of the robot [18]. The article highlights the methodology for evaluating legibility subjectively using specific questions and statements, and objectively by analyzing motion behavior, using metrics to evaluate the efficiency of interactions. The gradual analysis is closely related to human information processing [63]. Thereby, classic HRI evaluation methods are adapted to the domain of HRSI. As opposed to Hall et al. [17], who propose concentric circles as human-sensitive fields for static humans, Lam et al. [30] and Tomari et al. [59], who respectively propose egg-shaped and elliptical human-sensitive fields for HRSI and moving humans, the cone-shaped and head-orientation-fixed model IPS that Kitazawa and Fujiyama [26] proposed for interpersonal pedestrian interaction is applied, since it fits to moving pedestrians, the idea of a collision-free interaction [30], and the utilization of complementary head movements to perceive the visual field [14]. Accurate tracking and precise motion data of humans is necessary for high-performance robot control $[30,38]$. This aspect is accounted for by deriving highly frequent human motion data through the use of Vicon Nexus camera-based motion tracking. Results from this article may therefore serve as input for developers of robot control algorithms.

\section{VIDEO STUDY}

\subsection{Objective}

To target research question (1), an unbiased evaluation of plausible MCs is carried out by means of interviews. A team of three students designed three MC alternatives to a stop-and-wait strategy, each one of them intended to feasibly communicate "yielding priority" to an interacting person in front of a door. As a fundamental requirement, first an MC has to be perceived sensorily by a pedestrian [63], and subsequently an intention may be inferred [9]. These two processes are the focus of the video study. Subsequent response selection and response execution are targeted by the later motion tracking experiment.

\subsection{Study Design and Procedure}

In four videos, the viewer can see a door from a third-person perspective (Figure 3). From the left side, a person (student actor) moves toward the door. From the right side, a mobile robot (BEAM) approaches the door. In front of the door, cooperation between the person and the robot is initiated through one of the four robot MCs: stop (ST), back-off (BO), rotation (RO), and backoff and rotation (BORO). A roughly $90^{\circ}$ angle between the robot's path and the viewer of the video was applied to account for a viewpoint-based legibility optimization through undistorted motion [40]. The fixed third-person perspective is preferred to a head-mounted wide-angle camera, because wide-angle lenses lead to aberration errors, yet the view cannot be mapped to an individual person's view. One video per participant was presented on a tablet computer two times, including one of the four movement strategies as independent between-subject variable. As a dependent variable, two subsequent questions or statements were answered by the interviewees:

(1) "Please describe the robot's behavior."

(2) "Why did the robot show this behavior?"

The purpose of question (1) is to evaluate whether participants can describe relevant parameters of the movement. The assumption is that if they achieve that, they are able to observe and process the movement sensorily. With question (2), participants are asked why the robot showed the corresponding behavior, to analyze the intention participants derive from the robot. Open 

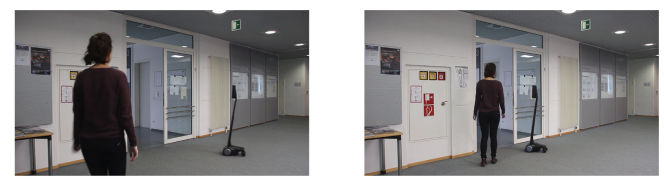

(a) First part of the videos: The robot drives toward the door.

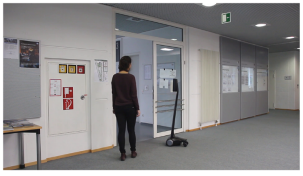

(b) ST: The robot stops and waits for $3 \mathrm{~s}$ before the video ends.

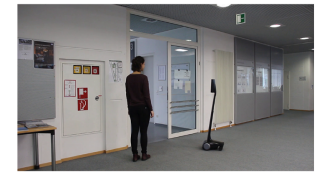

(c) BO: The robot displays a $3 \mathrm{~s}$ movement backward.

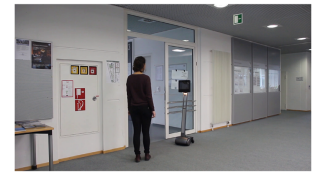

(d) RO: The robot rotates toward the pedestrian within $3 \mathrm{~s}$.

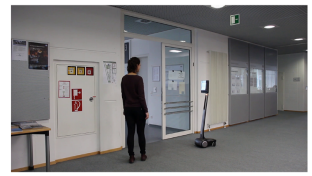

(e) BORO: The robot displays a 3s movement backward while rotating toward the pedestrian.

Fig. 3. Study design of the video study. The first part of the videos is equal for every group. The robot drives toward the door (a). After coming to a stop, the robot displays one of four different movement strategies aimed to solve the same task, to yield priority to the pedestrian. Video study pictures of the end positions of between-subject MCs, ST, BO, RO, and BORO, are displayed in (b) through (e).

questions are chosen to trigger unbiased responses about the qualitative intention. Using Likert scales, for example, would create a bias over the expected result of the interview. The questions were answered in the form of verbal interviews that were recorded and then transcribed.

\subsection{Sample}

In total, 167 participants ( 65 female, 102 male) were interviewed in this study, ranging from 17 to 78 years, with a mean age of 32.09 years $(S D=16.41$ years). Interviewees were acquired at two places. A student collective was acquired at the campus of the Technical University of Munich. The student collective involved 92 participants (23 female, 69 male) with ages ranging from 17 to 31 years and a mean of 21.81 years $(S D=2.72$ years). A public collective was interviewed in the Munich city center, at a subway station, and a shopping mall. The public collective consisted of 75 participants (42 female, 33 male) with ages ranging from 17 to 78 years and a mean of 44.69 years $(S D=17.40$ years). Selected at random, a participant received one of the four videos, resulting in a group distribution of $\mathrm{N}_{\mathrm{ST}}=43, \mathrm{~N}_{\mathrm{BO}}=42, \mathrm{~N}_{\mathrm{RO}}=43$, and $\mathrm{N}_{\mathrm{BORO}}=39$. In the public collective, $84.0 \%$ of interviewees answer that they had no previous experience with robots. In the student collective, $47.8 \%$ had no previous experience with robots. Participants received no payment for the interviews.

\subsection{Data Analysis}

A content analysis was applied to the acquired transcriptions. Text segments were categorized according to Hussy et al. [23]. Eight categories were composed of between two and five subcategories. Two raters (students) categorized the interviewees' answers into one of the subcategories. To tackle inter-rater reliability, a third rater was required if no agreement occurred between the two raters. For the analysis of research question (1), two categories are referred to in this article:

(1) Description of movement

(a) Correct, (b) Correct but incomplete, (c) Wrong, (d) No comment

(2) Recognition of robot intention

(a) To yield priority to the pedestrian, (b) To wait for the pedestrian, (c) Other intention, (d) No comment 
Description of movement

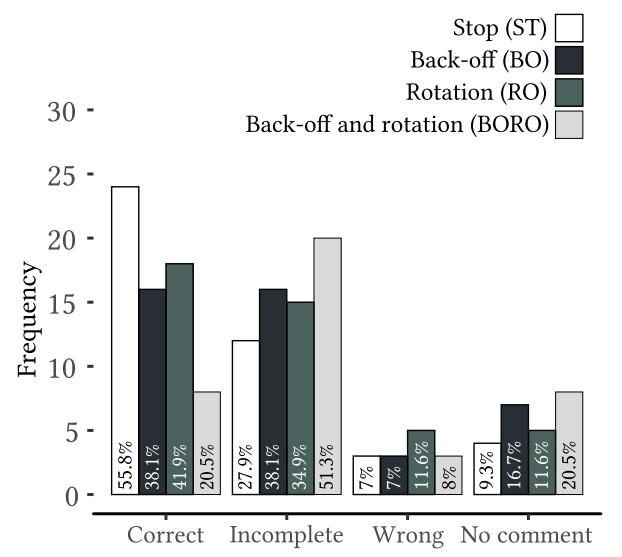

Fig. 4. Number of counts of individual participants' responses in the video study category (1), description of movement. Percent values indicate the percentage in the MC group of $\mathrm{N}_{\mathrm{ST}}=43, \mathrm{~N}_{\mathrm{BO}}=42, \mathrm{~N}_{\mathrm{RO}}=43$, and $\mathrm{N}_{\mathrm{BORO}}=$ 39.
Recognition of robot intention

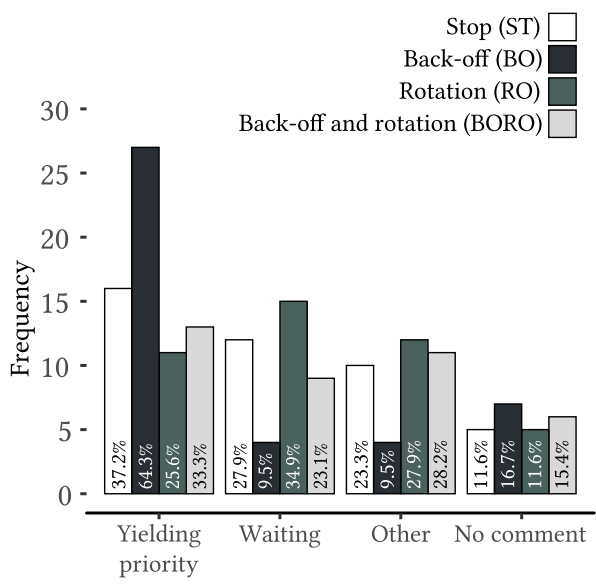

Fig. 5. Number of counts of individual participants' responses in the video study category (4), recognition of robot intention. Percent values indicate the percentage in the $\mathrm{MC}$ group of $\mathrm{N}_{\mathrm{ST}}=43, \mathrm{~N}_{\mathrm{BO}}=42, \mathrm{~N}_{\mathrm{RO}}=43$, and $\mathrm{N}_{\text {BORO }}=39$.

\subsection{Results}

Irrespective of the two collectives, public and student, the distribution of descriptions of the movement behavior (category 1) by participants are displayed in Figure 4. The distribution of the way interviewees recognized an intention (category 2) are displayed in Figure 5.

An investigation into the answers to recognition of robot intention (category 2) separated by the two interviewed collectives, irrespective of the $\mathrm{MC}$, reveals that $36 \%$ of the public collective recognizes the intention of yielding priority to the human, compared to $43.5 \%$ in the student collective. Additionally, $28.0 \%$ of the public collective responds with other intentions, compared to $17.4 \%$ in the student collective.

Compared to the other MCs, the $\mathrm{BO}$ was recognized as yielding priority to the humans by almost twice as many people in the sample as the ST (64.3\% compared to $37.2 \%)$. The result confirms that this MC may be further investigated and included in the main study. For further implications of the video study, please refer to the discussion in Section 4.1 of this article.

\section{MOTION TRACKING EXPERIMENT}

\subsection{Objective}

To target research question (2), a motion tracking experiment was conducted. The purpose of the experiment was to investigate whether the tendency to better identify the intention of "yielding priority" from a robot's BO, as opposed to merely a ST, would also lead to advantages in human efficiency while solving a cooperative situation in front of a bottleneck. A real encounter between uninformed pedestrians and a robot was planned to collect objective data in order to investigate differences in the behavior of participants. The agents should appear at the bottleneck at a similar time so that cooperation has to take place. When pedestrians infer the robot's intention quickly and correctly, we expect them to move through the bottleneck with a higher efficiency. If inferring takes a lot of time, we expect participants to slow down considerably, or come to a standstill. 


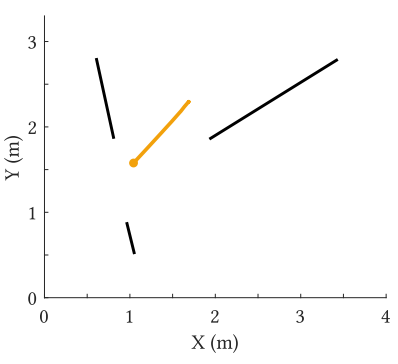

(a) Path of ST

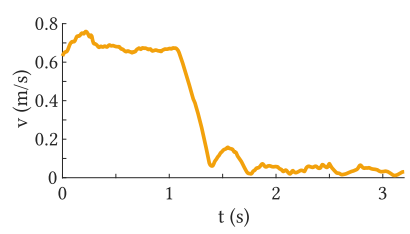

(d) Speed of ST

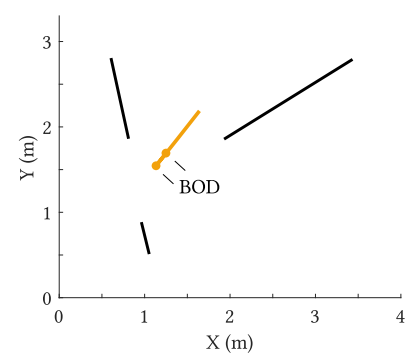

(b) Path of $\mathrm{BO}_{\text {short }}$

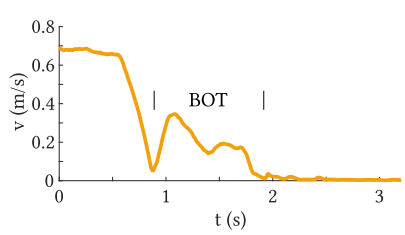

(e) Speed of $\mathrm{BO}_{\text {short }}$

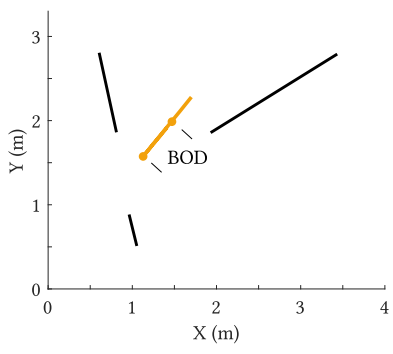

(c) Path of $\mathrm{BO}_{\text {long }}$

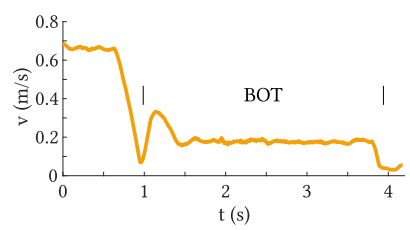

(f) Speed of $\mathrm{BO}_{\text {long }}$

Fig. 6. Three MCs, $\mathrm{ST}, \mathrm{BO}_{\text {short }}$, and $\mathrm{BO}_{\text {long }}$, are displayed in terms of two-dimensional path $(\mathrm{a}-\mathrm{c})$ and speed over time (d-f) diagrams. Dots in the trajectories (yellow) in (b) and (c) indicate positions where the robot came to a stop before and after the BO movement. The distance between the two points is called $B O D(\mathrm{~b}, \mathrm{c})$. The BOT can be retrieved from (e) and (f). Black lines in (a) through (c) indicate obstructions.

\subsection{Study Design}

We varied the robot's motion in three ways. The robot could perform a simple ST, a back-off short ( $\left.\mathbf{B O}_{\text {short }}\right)$, and a back-off long $\left(\mathbf{B O}_{\text {long }}\right.$ ) (Figure 6). The short and long versions of $\mathrm{BO}$ were designed to investigate whether the trajectory of driving backward would have an influence on intention inference and efficiency. These variations were motivated by the findings of Pacchierotti et al. [42], in which evasion distance was a matter of personal preferences and too wide an evasion could be perceived as inappropriate, and Kruse et al. [29], in which too small tweaks in the robot's motion caused confusion to the participants. There may be a range of back-off distances (BODs) that sufficiently communicate the intention of yielding priority, and the definition of an optimal maneuver can be critical [42]. Pacchierotti et al. [42] define three main parameters for describing and varying robotic movement, which are adapted for the design variations and the movement description in this study:

(1) The forward speed of the robot during the approach toward the door

(2) The distance the robot drives backward, BOD

(3) The time the robot spends driving backward, back-off time (BOT)

The perceivable robot kinematics are also defined by other, nonvaried parameters, such as accelerations or jerk, which are typically subject to the robot's capabilities and a result of mass inertia or engine power. In particular, the speed during the BO movement is not a design parameter in this study. It is limited by the experimental robot BEAM (Suitable Technologies, specifications: $1.34 \mathrm{~m} \times 0.22 \mathrm{~m} \times 0.42 \mathrm{~m}, 17.7 \mathrm{~kg}$ ) to a maximum of $0.2 \mathrm{~m} / \mathrm{s}$. In addition, accelerations and jerk cannot practicably be varied with this robot. Usually, this telepresence robot is steered with arrow keys or computer mouse via a desktop interface. Here, the three MCs are programmed as scripted movements using the software AutoHotkey to generate equal movements for all participants. Human-like robot speed is considered crucial for human interpretation in robot proximity 


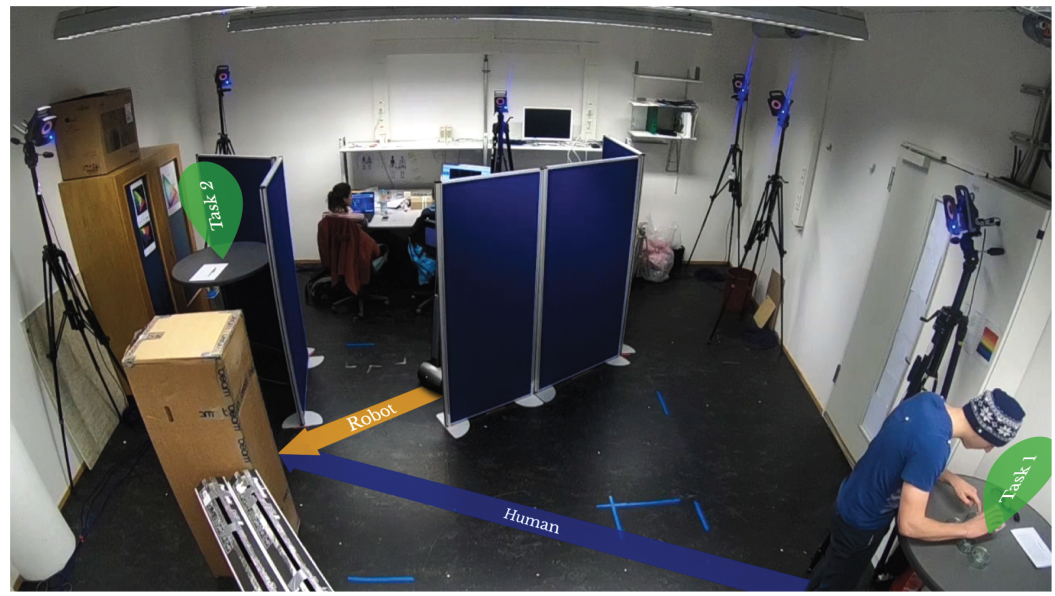

Fig. 7. Experimental space showing the estimated human path (blue) and robot path (yellow), the obstructions to form a bottleneck on the left (blue and cardboard), where the encounter between human and robot is planned, a visual obstruction between human and robot in the middle (blue), and the places for tasks 1 and 2 (green).

$[5,46]$. Accordingly, we aimed to achieve human-like speeds for an indoor environment. In any case, it should be higher than the speed used in Pacchierotti et al. [42], where the speed of $0.6 \mathrm{~m} / \mathrm{s}$ for an indoor robot was rated as too slow. For all MCs, the robot drives toward the bottleneck at approximately $0.7 \mathrm{~m} / \mathrm{s}$ before slowing down with a close to linear time-speed curve. In the ST condition, the robot simply stops and waits. In the $\mathrm{BO}_{\text {short }}$ condition, the robot drives backward for about back-off time $(\mathrm{BOT})_{\text {short }}=1 \mathrm{~s}$ and thereby reaches a back-off distance $(\mathrm{BOD})_{\text {short }}=0.19$ $\mathrm{m}$ before coming to a stop again. In the $\mathrm{BO}_{\text {long }}$ condition, the robot drives backward for about $\mathrm{BOT}_{\text {long }}=3 \mathrm{~s}$ and thereby reaches a $\mathrm{BOD}_{\text {long }}=0.54 \mathrm{~m}$. The $\mathrm{BO}_{\text {long }}$ applied in the motion tracking experiment is equal to the $\mathrm{BO}$ used in the video study. To investigate a learning curve while managing experimentation time, a total of three encounters with the MCs was planned.

The experimental space was set up inside the motion lab of the Chair of Ergonomics (Figure 7). Obstructions were placed to create a bottleneck inspired by real-world door widths in Germany of $0.95 \mathrm{~m}$ (DIN 18040-1), and additional obstructions were placed to inhibit visual contact between participants and the mobile robot before the encounter. The distance between the center of the bottleneck and the visual obstruction measured $1.15 \mathrm{~m}$. Participants had no secondary task while walking. Instead, participants had to alternate between two tasks at either side of the bottleneck. In task 1, washers had to be sorted into bins according to their size. During task 2, simple mathematical calculations had to be solved. This was applied to counteract participants following up on unpredictable secondary tasks, and instead find a common task that can be observed by the experimenter and does not pose immediate harm to awareness while interacting with the robot [18]. Participants were not informed a priori about the appearance of a robot during the study. A participant should only experience one of the three MCs to avoid confounding the effect with various movement alternatives and the assumption that robots should ultimately show consistent behavior for fluent and recognizable interaction [45]. Accordingly, three trials are designed as within-subject variable, and the three MCs as the between-subject variable, resulting in a $3 \times 3$ mixed study design. Eight Vicon Nexus cameras were installed for motion tracking, and infrared light reflecting markers were placed on the participants' clothes. Two statements about the first encounter with the robot were applied to acquire additional subjective feedback about the robot's 
Table 1. Study Procedure of the Motion Tracking Experiment Only Including Task Switches from Task 1 to Task 2 and According Robot Behavior: Between-Subject MCs; ST, BO $\mathrm{BO}_{\text {short }}, \mathrm{BO}_{\text {long, Which }}$ Are the Trials T1, T2, and T3; Task Switches Where Not Robot Appeared; and One Task Switch Where the Robot Passes the Bottleneck Before the Participant

\begin{tabular}{ll}
\hline Human Switch from Tasks 1 to 2 & \multicolumn{1}{c}{ Robot Behavior } \\
\hline 1 & MC (trial 1), the robot passes the bottleneck after the participant \\
2 & The robot passes the bottleneck before the participant \\
3 & No robot appears \\
4 & MC (trial 2), the robot passes the bottleneck after the participant \\
5 & No robot appears \\
6 & MC (trial 3), the robot passes the bottleneck after the participant \\
\hline
\end{tabular}

intention using a 5-point Likert scale ranging from strongly disagree to strongly agree (or no answer). These statements were applied at the end of the experiment to prevent a bias:

(1) "The robot wanted to let me pass."

(2) "The robot made its intentions clear."

\subsection{Procedure}

Upon starting the experiment, participants were introduced to their tasks. An audible signal (beep tone) instructed participants to stop the current task after $20 \mathrm{~s}$ and walk through the bottleneck to begin the other task. Six task switches from task 1 to task 2 and six switches back from task 2 to task 1 had to be performed. Participants were assured that the time spent in between the two tasks would not influence their overall performance. Additionally, they were instructed to move at a comfortable walking speed. In the analysis, only task switches from task 1 to task 2 are considered (Table 1). When participants started to walk toward the bottleneck while switching from task 1 to task 2 for the first, fourth, and sixth time, the robot appeared from behind the obstruction initiated by the experimenter, and showed an MC, resulting in trial 1 (T1), trial 2 (T2), and trial 3 (T3). In these task switches, the robot was moved through the bottleneck after the participant and was then turned around to drive back through the bottleneck to its initial position while the participant solved task 2 . This was performed manually by the experimenter. In the second task switch, the robot drove through the door in a dominant manner, not yielding priority to the participant. This section was included to give the participants the impression that robots can show variable behavior and not only act submissively, so subsequent encounters could still exclusively embrace the effect of motion legibility. Humans can quickly activate a mental model and the impression of robotic action could shift toward anticipated instead of legible behavior [56]. In task switches three and five, no robot appeared. In principle, the robot did not appear at the bottleneck when participants walked from task 2 to task 1 . The robot's screen had no content and was left black. At the end of the experiment, participants were reminded to imagine their first encounter with the robot and answered the statements. The experiment took approximately 15 minutes. Nine minutes were spent on explanation, labeling for motion tracking, and the questionnaire. Six minutes were spent on the tasks and switches.

\subsection{Sample}

The experiment sample consisted of 78 participants (28 female, 50 male) ranging from 16 to 29 years, with a mean age of 21.83 years $(S D=2.56$ years), acquired at the campus of the Technical University of Munich. Excluded were persons that had taken part in the video study. The 


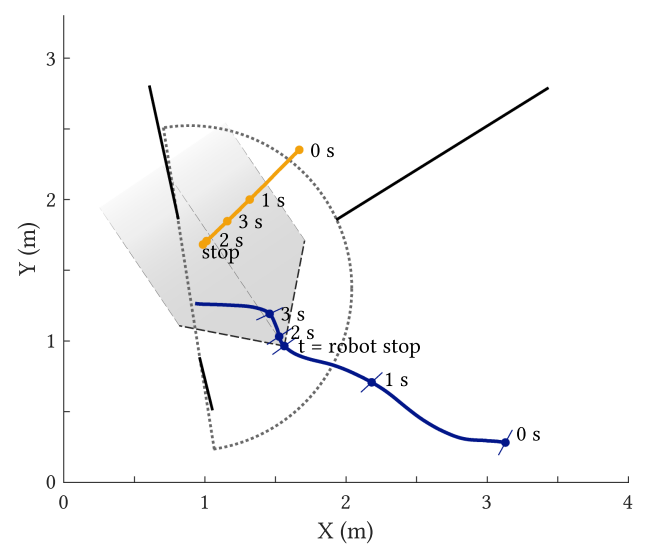

(a) Valid encounter of participant 6 in trial 1

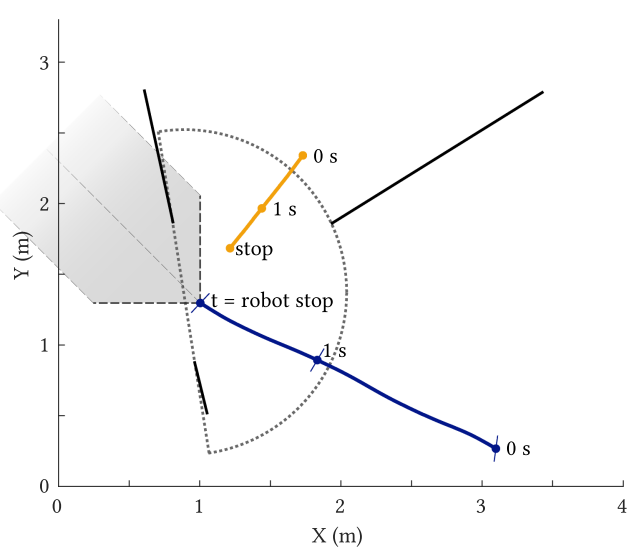

(b) Invalid encounter of participant 37 in trial 1

Fig. 8. Trajectories of the human head (blue) and robot (yellow) are displayed with dots marking positions at timesteps of $1 \mathrm{~s}$. The IS is displayed as a semicircle (dotted). The human head orientation is perpendicular to the vector from LEAR to REAR at every position (thin blue lines). In (a), the robot comes to a stop inside the human IPS (grey) at $t=$ robot stop, and in (b), the robot is outside the IPS at $t=$ robot stop. Black lines indicate obstructions.

collective was split into three groups by order of acquisition, resulting in an equal group distribution of $\mathrm{N}=26$ for each group. Of the participants, $25.9 \%$ answered that they had no previous experience with robots. Participants received no payment and did not suffer from any movement disorders or other mobility limitations.

\subsection{Data Analysis}

Motion data of the robot and participants was captured using the Vicon Nexus with a capture rate of 120 fps. Infrared-light-reflecting markers were placed on the participants' shoes, legs, left shoulder, right shoulder, clavicle (CLAV), left ear (LEAR), and right ear (REAR). Additionally, an imaginary marker was calculated as a mean point between the left ear (LEAR) and right ear (REAR) to retrieve data for the center of the head. The head orientation can be calculated as perpendicular to the unit vector from LEAR to REAR. The robot position was represented by a marker placed at the front of the base of the robot. Data was analyzed in the two-dimensional X-Y plane only. Sometimes, tracking was lost in the raw data. Human clavicle (CLAV) data loss accounted for $M_{\mathrm{T} 1}=14.00 \%\left(S D_{\mathrm{T} 1}=24.16 \%\right), M_{\mathrm{T} 2}=13.06 \%\left(S D_{\mathrm{T} 2}=24.47 \%\right)$, and $M_{\mathrm{T} 3}=12.03 \%\left(S D_{\mathrm{T} 3}=22.74 \%\right)$ of data points. The lost data is reconstructed by linear interpolation assuming a constant speed of the lost markers inside the gap. In accordance with Schmidtler et al. [51], a 5-point stencil is applied to derive velocity data from the CLAV position data, where $h$ is one step in the data (Equation (1)).

$$
v_{(x)} \approx \frac{-p_{(x+2 h)}+8 p_{(x+h)}-8 p_{(x-h)}+p_{(x-2 h)}}{12 h}
$$

The interaction space (IS) is introduced to the experimental space (Figure 8). It is computed in the shape of a semicircle. The flat side is defined by the obstructions of the bottleneck. The circular side has a radius of $1.15 \mathrm{~m}$, which is the distance between the middle of the bottleneck and the visual obstruction. The interaction space (IS) is used to compute and compare motion data for measures of efficiency, from when the participant enters the IS until he or she passes the bottleneck. It is assumed that humans could have visual contact with the robot (have passed 
the visual obstruction) and are in equal proximity to the door. Furthermore, it should begin at a distance to task 1 , where participants have already reached walking speed after accelerating from a standing position [61]. A comfortable walking speed for 20- to 30-year-old women is $M=1.41 \mathrm{~m} / \mathrm{s}$ $(S D=0.18 \mathrm{~m} / \mathrm{s})$, and men reach $M=1.39 \mathrm{~m} / \mathrm{s}(S D=0.15 \mathrm{~m} / \mathrm{s})$ [3]. According to Muir et al. [39], 20 - to 25-year-old pedestrians reach their self-selected gait speed roughly three steps after gait initiation. Derived from the investigations by Jian et al. [24], a typical step length is estimated as $0.5 \mathrm{~m}$. The distance between the start of the observable area (motion tracking starts where $\mathrm{t}=0 \mathrm{~s}$ in Figure 8) and the outer limit of the IS measures approximately $1.5 \mathrm{~m}$ in the scenario at hand. Given that the place of task 1 is actually situated farther right than the start of the observable area, the experimental space allows participants to reach their comfortable walking speed before entering the IS.

For the scope of this experiment, a valid statement about the legibility of an $\mathrm{MC}$ requires that a participant and the robot arrive at the bottleneck at roughly the same time, so a need to arrange an order of passage exists [61]. Some of the encounters between human and robot do not work out as planned by the experimenter. Despite being instructed otherwise, sometimes participants start to walk too early, ahead of the beep tone. The robot's movement has to be initiated by the experimenter and cannot be executed in time, resulting in participants passing the bottleneck too early without experiencing the MCs. In other cases, participants walk too slowly and the robot reaches the door too early, displaying an MC that is still out of sight of the participants. To identify an encounter as a valid encounter, it is a necessary requirement that the participant is able to see the robot's MC and considers the robot an obstacle. To account for this, the IPS is applied to a human head-oriented coordinate system. We formulate an algorithm that evaluates an encounter as valid if the robot is inside the IPS at the moment the robot stops (a robot stop occurs when its velocity reaches $\mathrm{v}<0.1 \mathrm{~m} / \mathrm{s}$ ), just before displaying the $\mathrm{MC}$, and invalid otherwise (see Figure 8). Moments when the robot is hidden behind the visual obstruction and therefore out of sight for the participant are similarly considered invalid.

To account for misleading head rotations, an additional visual inspection of the video recordings of the excluded encounters is performed. This leads to the reconfirmation of three encounters in $\mathrm{T} 1$, seven encounters in T2, and three encounters in T3 that were previously excluded by the algorithmic approach. It is possible that participants rotate the head away from the robot just moments before it comes to a stop, yet the robot is inspected before and after this event. Additionally, eye movement does not necessarily follow head rotation [14]. Visual inspection of the recordings in these cases confirms that a conscious encounter actually took place. In total, 13 of 234 encounters $(5.56 \%)$ are handled this way. Considering a trial of an individual participant is only valid when all previous trials of the respective participant are valid, 18 valid encounters are left in the ST condition in $\mathrm{T} 1,14$ in $\mathrm{T} 2$, and 13 in T3. For $\mathrm{BO}_{\text {short }}, 19$ valid encounters are left in T1, 17 in T2, and 15 in $\mathrm{T} 3$. For $\mathrm{BO}_{\text {long }}, 21$ valid encounters are left in T1, 18 in T2, and 18 in T3. For statistical analysis, an individual participant is only included when all trials of respective participant are valid (in this case, a participant has experienced the full experiment). Accordingly, the dataset is reduced to 13 participants for MC ST, 15 for $\mathrm{BO}_{\text {short }}$, and 18 for $\mathrm{BO}_{\text {long. }}$.

\subsection{Results}

3.6.1 Time for Interaction. To measure efficiency, we calculate the time participants need between entering the IS and passing the bottleneck and leaving the IS, the time for interaction (TFI). A $3 \times 3$ two-factorial mixed design ANOVA with MC as a between factor of three levels and trial as within factor of three levels is applied to analyze the effect of $\mathrm{MC}$ on time for interaction $(\mathrm{TFI})\left(F(2,43)=0.95, p=0.39, \eta^{2}\right.$ partial $\left.=0.0423\right)$ and trial on TFI $(F(2,86)=4.97, p=0.009$, $\eta^{2}$ partial $\left.=0.1036\right)$. The TFI decreases over trials with a statistical significance $(p<0.05)$. Figure 9 


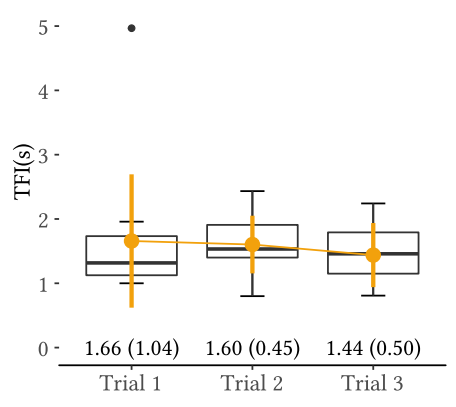

(a) TFI in the ST group $(\mathrm{N}=13)$

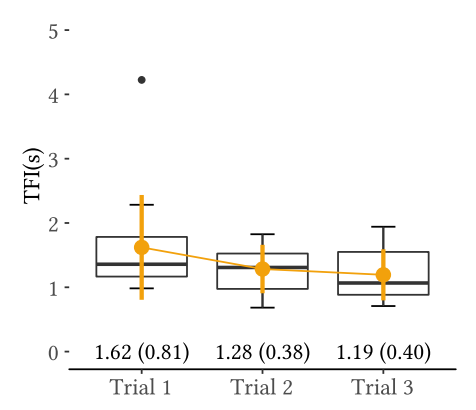

(b) TFI in the $\mathrm{BO}_{\text {short }}$ group $(\mathrm{N}=15)$

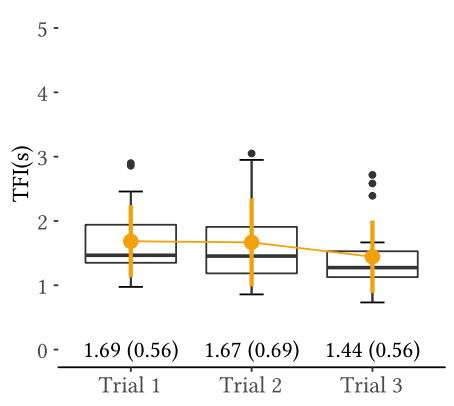

(c) TFI in the $\mathrm{BO}_{\text {long }}$ group $(\mathrm{N}=18)$

Fig. 9. The resulting TFI according to three MCs and three trials. Yellow dots mark the mean for each condition. Yellow lines display the standard deviation. Corresponding values are displayed at the bottom of the plot (standard deviation in brackets). Boxplots are visualized in the background of the plot (black).

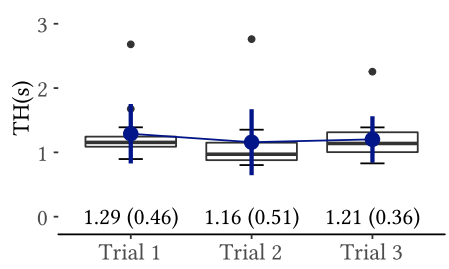

(a) $\mathrm{TH}$ in the ST group $(\mathrm{N}=13)$

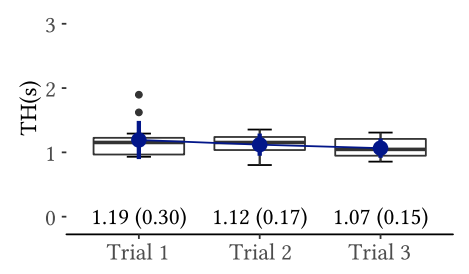

(b) $\mathrm{TH}$ in the $\mathrm{BO}_{\text {short }}$ group $(\mathrm{N}=15)$

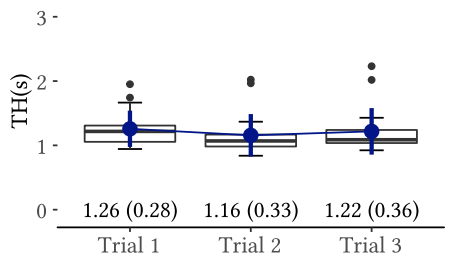

(c) $\mathrm{TH}$ in the $\mathrm{BO}_{\text {long }}$ group $(\mathrm{N}=18)$

Fig. 10. The resulting TH according to three MCs and three trials. Blue dots mark the mean for each condition. Blue lines display the standard deviation. Corresponding values are displayed at the bottom of the plot (standard deviation in brackets). Boxplots are visualized in the background of the plot (black).

provides TFI separated for each MC and trial. There are no significant differences between the ST and the $\mathrm{BO}_{\text {short }}$ group $(p=0.06)$ and between the ST and $\mathrm{BO}_{\text {long }}$ group $(p=0.23)$ in post hoc contrast analysis, irrespective of the trial. The corresponding test for differences in trials reveals $p$-values between $\mathrm{T} 1$ and $\mathrm{T} 2$ of $p=0.89$ and between $\mathrm{T} 1$ and $\mathrm{T} 3$ of $p=0.04$. The latter comparison is statistically significant, which means that TFI was lower in T3 compared to T1, irrespective of the MC. Accounting for MC and trial in post hoc contrast analysis, participants need significantly less time in $\mathrm{T} 2$ in the $\mathrm{BO}_{\text {short }}$ group than in $\mathrm{T} 2$ in the $\mathrm{ST}$ group $(p=0.04)$.

3.6.2 Human Time. To evaluate the real implications of the encounter on humans, the time that pedestrians typically need when there is no robot, the time by humans (TH), is investigated. The mean walking speed of each participant during the $0.5 \mathrm{~s}$ before entering the IS is calculated, and a constant speed over the distance of the IS $(1.15 \mathrm{~m})$ is assumed. Speeds calculated for the sample range from $M_{\mathrm{BO}_{\text {long }} 1}=0.95 \mathrm{~m} / \mathrm{s}\left(S D_{\mathrm{BO}_{\text {long }} \mathrm{T} 1}=0.18 \mathrm{~m} / \mathrm{s}\right)$ to $M_{\mathrm{BO}_{\text {short }} \mathrm{T} 3}=1.10 \mathrm{~m} / \mathrm{s}$ $\left(S D_{\mathrm{BO}_{\text {short }} \mathrm{T} 3}=0.16 \mathrm{~m} / \mathrm{s}\right)$. With these speeds, the time through the IS without encountering a robot in respective trials is estimated. Figure 10 shows the resulting time by humans (TH). Mean times do not vary much, and an ANOVA shows no significant differences for $\operatorname{MC}(F(2,40)=0.37, p=0.69$, $\left.\eta_{\text {partial }}^{2}=0.0183\right)$ and trial $\left(F(2,80)=2.71, p=0.07, \eta^{2}\right.$ partial $\left.=0.0635\right)$.

3.6.3 The Robot's Influence. To display the influence the robot had on each participant, the individual TH is subtracted from the individual TFI. Figure 11 shows the resulting TFI - TH. 


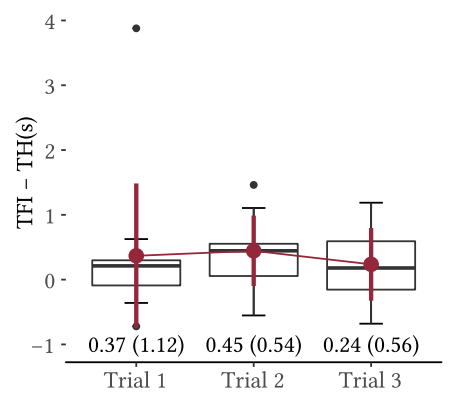

(a) TFI - TH in the $\mathbf{S T}$ group $(\mathrm{N}=13)$

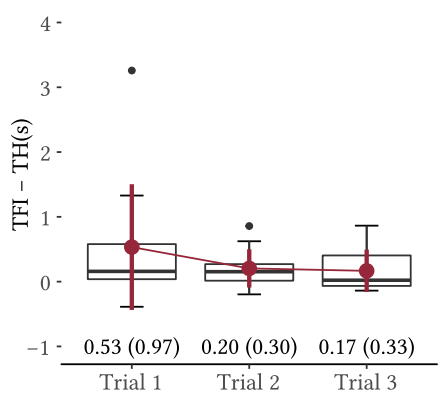

(b) TFI - TH in the $\mathbf{B O}_{\text {short }}$ group $(\mathrm{N}=15)$

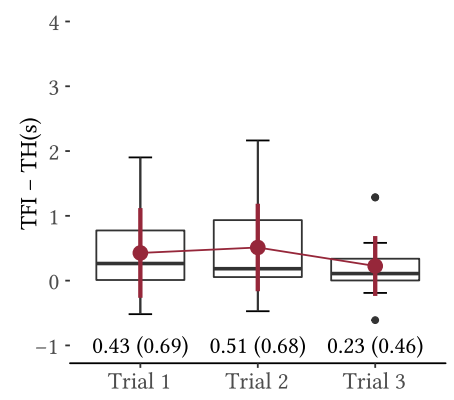

(c) TFI - TH in the $\mathbf{B O}_{\text {long }}$ group $(\mathrm{N}=18)$

Fig. 11. The resulting TFI - TH according to three MCs and three trials. Red dots mark the mean for each condition. Red lines display the standard deviation. Corresponding values are displayed at the bottom of the plot (standard deviation in brackets). Boxplots are visualized in the background of the plot (black).

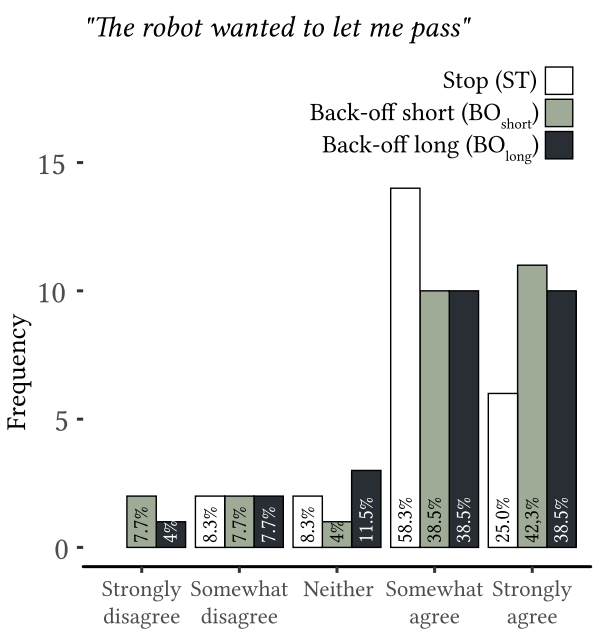

Fig. 12. Number of counts of individual participants' responses in the statement "The robot wanted to let me pass." Percent values indicate the percentage in the MC group of $\mathrm{N}=24(\mathrm{ST})$, $\mathrm{N}=26\left(\mathrm{BO}_{\text {short }}\right)$, and $\mathrm{N}=26\left(\mathrm{BO}_{\text {long }}\right)$ participants. Where the sample size differs from 26 , missing participants gave no answer.

\section{"The robot made its intentions clear"}

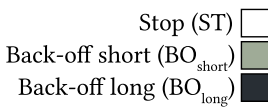

$15-$

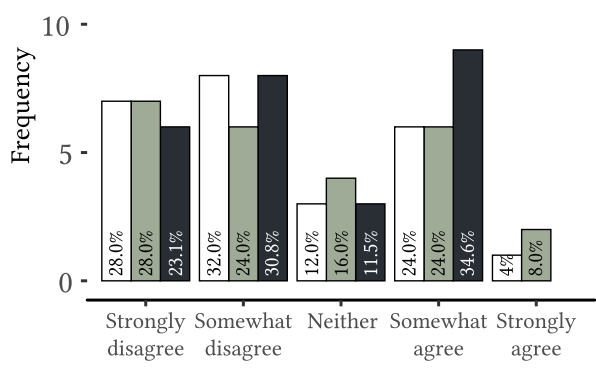

Fig. 13. Number of counts of individual participants' responses in the statement "The robot made its intentions clear." Percent values indicate the percentage in the $\mathrm{MC}$ group of $\mathrm{N}=25$ (ST), $\mathrm{N}=25\left(\mathrm{BO}_{\text {short }}\right)$, and $\mathrm{N}=26$ ( $\left.\mathrm{BO}_{\text {long }}\right)$ participants. Where the sample size differs from 26, missing participants gave no answer.

No significant differences appear for $\operatorname{MC}\left(F(2,40)=0.10, p=0.91, \eta^{2}\right.$ partial $\left.=0.0047\right)$ or for trial $\left(F(2,80)=2.50, p=0.09, \eta^{2}\right.$ partial $\left.=0.0588\right)$.

3.6.4 Subjective Evaluation. The distribution of answers to the statements "the robot wanted to let me pass" and "the robot made its intention clear" ranging from strongly disagree to strongly agree are displayed in Figure 12 and Figure 13.

\section{DISCUSSION}

To fluently interact with a robot's motion strategy, a pedestrian engages with sensory and cognitive perception of the behavior, and the selection and execution of a suitable response [63]. To ease this, 
motion legibility of a BO MC is given if human reactions show that the robot's intention is inferred confidently and a cooperative scenario between human and robot can be solved efficiently $[9,20]$.

\subsection{Implications of the Video Study}

Motivated by related works and our own previous experiments, the goal of this article's first experiment is to evaluate human sensory perception and the subjective legibility of a robot's BO compared to three other motion strategies, ST, RO, and BORO, in a video study at the university campus and public spaces with $\mathrm{N}=167$ interviewees. Results indicate that $\mathrm{BO}$ excels in legibility and is correctly (38.1\%) or correctly and merely incompletely (38.1\%) described, and thus perceived sensorily by the majority of interviewees.

Heterogeneous descriptions of the robot's movement need further investigation. Although the majority of interviewees infer the correct intention of $\mathrm{BO}$, a great deal of participants incompletely describe this movement. The ST is described more precisely. We assume that the general complexity of the movement is low in the ST movement. Interviewees are less prone to make mistakes when describing the stop of a robot in comparison with a robot that first stops, then backs-off, and comes to a halt again (or performs an additional rotation). This could have led to the higher number of answers that incompletely describe BO, RO, and BORO compared to ST. To be labeled a correct description of the $\mathrm{BO}$ behavior, the answer has to include features such as "[...] it drives toward the door [...], then it stops for a moment and then it drives back again and waits," whereas an incomplete answer leaves out details such as "robot drives to the door, [...] slows down and makes room."

Intention recognition by older interviewees at public spaces was more heterogeneous. A followup study therefore may target older pedestrian groups specifically. It should be noted that the videos were arranged to view the robot from a third-person perspective to give a constant perspective of the robot's movement. It is not clear what role the third-person view and, thus being an actor excluded from the HRI, plays in the statements by the interviewees. Deliberately, the video study had a qualitative character, making it applicable to short interview duration and a representative public collective. The video study's conclusion that the $\mathrm{BO}$ is suitable for communicating the intention of yielding priority to pedestrians at bottlenecks led to its application in the main study of this article, the motion tracking experiment.

\subsection{Implications of the Motion Tracking Experiment}

Implemented in a real encounter, objective motion behavior of $\mathrm{N}=78$ participants as a reaction to a stop motion strategy $(\mathrm{ST})$ and two versions of $\mathrm{BO}\left(\mathrm{BO}_{\text {short }}\right.$ and $\left.\mathrm{BO}_{\text {long }}\right)$ shows a significant improvement in the pedestrians' efficiency in the second encounter with the robot's short $\mathrm{BO}$ version compared to the ST strategy. Thus far, $\mathrm{BO}_{\text {short }}$ allows for fastest learning in a sample of university students where the majority had some previous experience with robots.

Ultimately, participants interact efficiently with all of the motion strategies after three trials, since the temporal influence of the encounter is improved, leaving a small remaining time consumption that the cognitive process of perceiving any object in the visual field requires. The range of remaining TFI - TH in T3 from $M_{\mathrm{BO}_{\text {short }} \text { T3 }}=0.17 \mathrm{~s}$ to $M_{\mathrm{STT} 3}=0.24 \mathrm{~s}$ indicates that the robot does not have a vast impact in the third encounter. Yet some sort of temporal influence is always present when an interaction occurs. When an encounter with a robot is unplanned, humans need 0.4 to $1.0 \mathrm{~s}$ to scan the environment and to fixate the eyes on the robot $[55,62,64]$, which corresponds to

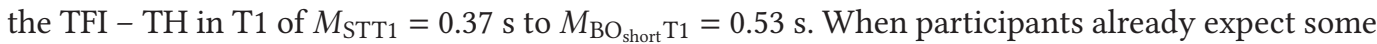
sort of interaction in later trials, a shift in perception from the visual field to the visual world may occur. Here, the expected TFI - TH may also be lower. 
Deploying such a HRSI experiment poses hurdles to initiate comparable encounters. The freedom of movement of pedestrians implies that no interaction is exactly the same. The debatable degree to which situations can be regarded as comparable influences reported results. In the presented study, the valid sample of participants is reduced by strict constraints to the validity of encounters. First of all, motion data of high quality were necessary for this analysis. The approximately $3 \times 4$ m experimental space was about the maximum that can be tracked with eight Vicon Nexus cameras. The obstructions placed, in particular, posed difficulties in that they inhibited the visibility of the infrared-reflecting markers to some extend. Through interpolation, a consistent dataset is achieved. When requiring the presence of the MC inside the human IPS to account for valid encounters, the theory of mental models by Sebanz et al. [56] is neglected to some extent. Mental models of the robot begin to exist in humans' minds while acquainting to the robot. After the first encounter, participants may anticipate the BO during $\mathrm{T} 2$ and $\mathrm{T} 3$ even though they do not perceive it sensorily in their IPS. This flaw was accounted for by reviewing video recordings of the excluded encounters for a second time. Additionally, the occasional dominant robot behavior should counteract the fast creation of an exact mental model. Only 13 of 234 total encounters $(5.56 \%)$ had to be revalidated after visual inspection. Thus, mental models seem to have minor impact at this early stage of acquainting to a robot. Accordingly, using the presence of the robot in the human IPS as measure for encounter validity can be regarded as a feasible method.

In subjective evaluations of the motion tracking experiment, both variations, $\mathrm{BO}_{\text {short }}$ and $\mathrm{BO}_{\text {long }}$ pose a stronger sense to communicate to let pedestrians pass, compared to ST. As such, $42.3 \%$ and $38.5 \%$ "strongly agree" with the corresponding statement in the two groups that experienced $\mathrm{BO}_{\text {short }}$ and $\mathrm{BO}_{\text {long, }}$, compared to merely $25.0 \%$ of participants in the ST group. Although many participants (58.3\%) somewhat agree with this statement in the ST group, the difference in participant numbers who "strongly agree" is apparent. In contrast, results are comparably heterogeneous regarding the statement "the robot made its intentions clear." Participants indicate rather low ratings with "strongly disagree" in $28.0 \%, 28.0 \%$, and $23.1 \%$ for $\mathrm{ST}, \mathrm{BO}_{\text {short }}$, and $\mathrm{BO}_{\text {long }}$, respectively, suggesting low legibility in all groups. These differences could be an effect of the generic wording. Despite being reminded to evaluate the first encounter, participants may have also included the mix with dominant robot behavior in their answers, resulting in mixed feelings about the clearness of intentions.

Similar to the presented study, Moon et al. [36] found less evidence in objective measures like human performance than in subjective measures, where participants regarded the movements as hesitations. The authors did not find sufficient evidence that the hesitation motion profile improved human-robot task completion time, whereas results suggested that participants can recognize such robot responses as hesitations and distinguished them from abrupt stopping behaviors.

In any case, collisions with robots have to be prevented (rule (1) [30]). We designed the experiment and the participants' tasks in a way that should allow attention to their surroundings and awareness of the robot. Virtually every participant answered subjective questions, and hence it could be assumed that everyone is aware of the robot. However, partially and unaware participants tend to reduce their cognitive dissonance by creating stories about a stimulus they had not seen [18], limiting the aforementioned assumption to some extent. In the study of Harms et al. [18], the absence of awareness does not imply an absence of perception and the risk of collisions with a static obstacle. For our scope, however, it is not clear to what extent walking without awareness in a natural public environment poses risks of collisions with a mobile robot. The difference between presented HRSI and the experiment by Harms et al. [18] becomes apparent in deviating numbers of participants assumed to be unaware, and the presence of a moving obstacle (robot). We argue that allowing interference-free navigation for pedestrians (rule (2) [30]) may be impossible when bottlenecks have to be passed by both actors in a cooperative scenario. Yet we showed that the 
Table 2. Additional Metrics for Three MCs; ST, BO $\mathrm{B}_{\text {short }}$, $\mathrm{BO}_{\text {long }}$, and Trials T1 - T3: Speed Reduction, Trajectory Energy, Minimum Distance, Idle Time

\begin{tabular}{lccccccccc}
\hline Metric & \multicolumn{3}{c}{$\mathrm{ST}$} & \multicolumn{4}{c}{$\mathrm{BO}_{\text {short }}$} & \multicolumn{3}{c}{$\mathrm{BO}_{\text {long }}$} \\
& $\mathrm{T} 1$ & $\mathrm{~T} 2$ & $\mathrm{~T} 3$ & $\mathrm{~T} 1$ & $\mathrm{~T} 2$ & $\mathrm{~T} 3$ & $\mathrm{~T} 1$ & $\mathrm{~T} 2$ & $\mathrm{~T} 3$ \\
\hline Speed reduction $(\mathrm{m} / \mathrm{s})$ & 0.73 & 0.91 & 0.70 & 0.77 & 0.68 & 0.50 & 0.76 & 0.86 & 0.67 \\
& $(0.35)$ & $(0.40)$ & $(0.41)$ & $(0.38)$ & $(0.29)$ & $(0.26)$ & $(0.34)$ & $(0.38)$ & $(0.37)$ \\
Trajectory energy $\left(\mathrm{m}^{2} / \mathrm{s}^{2}\right)$ & 0.84 & 0.88 & 0.93 & 0.80 & 0.79 & 1.00 & 0.83 & 0.90 & 0.93 \\
& $(0.15)$ & $(0.15)$ & $(0.19)$ & $(0.24)$ & $(0.37)$ & $(0.33)$ & $(0.12)$ & $(0.22)$ & $(0.24)$ \\
Minimum distance $(\mathrm{m})$ & 0.40 & 0.37 & 0.44 & 0.46 & 0.51 & 0.48 & 0.55 & 0.53 & 0.57 \\
& $(0.13)$ & $(0.12)$ & $(0.07)$ & $(0.17)$ & $(0.09)$ & $(0.10)$ & $(0.14)$ & $(0.14)$ & $(0.05)$ \\
Idle time (s) & 1.59 & 1.11 & 0.75 & 0.59 & 0.67 & 0.59 & 0.99 & 1.00 & 0.94 \\
& $(1.85)$ & $(0.22)$ & $(0.36)$ & $(0.29)$ & $(0.12)$ & $(0.40)$ & $(0.48)$ & $(0.59)$ & $(0.50)$ \\
\hline
\end{tabular}

Values show means (standard deviation in parentheses).

interaction can be handled in better and worse ways. A worst practice could be described with the participant of the ST group, who stands still and watches the standing robot for a crippling $3.80 \mathrm{~s}$ (TFI is approximately $4.97 \mathrm{~s}$ in Figure 9), before deciding to continue through the bottleneck, during the first encounter with the robot. At the current state of knowledge, we suggest to continue testing $\mathrm{BO}_{\text {short }}$ as an adaption to waiting rule (3) of Lam et al. [30] for legible robot behavior that has the potential to increase human efficiency. The presented methods for designing and evaluating legibility of motion strategies proved to give meaningful insights into the communicative quality of robot motion. These methods may be used and adapted for further variations of scenarios and different behavioral approaches for robots.

\subsection{Additional Metrics of Human Behavior}

Although we proposed time as a proxy for efficiency according to Steinfeld et al. [58], other authors include additional metrics as representative measures of human trajectory smoothness in their analysis of human-aware navigation algorithms [4,34]. Mavrogiannis et al. [34] find varying correlations of these metrics with navigation strategies in their experiments. In this section, we aim to give a broader perspective on human behavior and preference in our experiment by using the acquired position and velocity data to apply additional metrics in an explorative analysis.

Under the assumption that strong changes in velocity correlate with higher acceleration in a respective time frame, we adopt the considerations of average acceleration from Mavrogiannis et al. [34]. We calculate the difference of maximum speed before entering the IS and minimum speed inside the IS, the speed reduction (Table 2). A $3 \times 3$ two-factorial mixed design ANOVA with $\mathrm{MC}$ as a between factor of three levels and trial as within factor of three levels is applied to analyze the effect of MC on speed reduction $\left(F(2,40)=0.75, p=0.480, \eta^{2}\right.$ partial $\left.=0.0360\right)$ and trial on speed reduction $\left(F(2,80)=4.51, p=0.014, \eta^{2}\right.$ partial $\left.=0.1013\right)$. The speed reduction differs over trials with a statistical significance $(p<0.05)$. Post hoc contrast analysis for differences in trials reveals $p$-values between T1 and T2 of $p=0.046$ and between the T1 and T3 of $p=0.013$. The comparisons are statistically significant, which means that speed reduction is greater in T2 compared to T1 and lower in T3 compared to T1, irrespective of the MC. Accounting for MC and trial in post hoc contrast analysis, we do not find statistically significant differences between the $\mathrm{MC}$ groups within each trial. This means that there is no effect of the application of a specific MC on the way participants reduce their walking speed in the presented study.

To measure trajectory energy, we calculate the integral of the squared velocity over the trajectory while a person is inside the IS according to Mavrogiannis et al. [34] (see Table 2). A $3 \times 3$ 
two-factorial mixed design ANOVA with MC as a between factor of three levels and trial as within factor of three levels is applied to analyze the effect of $\mathrm{MC}$ on trajectory energy $\left(F(2,43)=0.08, p=0.924, \eta^{2}\right.$ partial $\left.=0.0037\right)$ and trial on trajectory energy $(F(2,86)=5.99, p=0.004$, $\eta^{2}$ partial $\left.=0.1223\right)$. The trajectory energy differs over trials with a statistical significance $(p<0.05)$. There are no significant differences between the ST and the $\mathrm{BO}_{\text {short }}$ group $(p=0.605)$ and between the $\mathrm{ST}$ and $\mathrm{BO}_{\text {long }}$ group $(p=0.808)$ in post hoc contrast analysis, irrespective of the trial. The corresponding test for differences in trials reveals $p$-values between T1 and T2 of $p=0.532$ and between the T1 and T3 of $p=0.009$. The later comparison is statistically significant, which means that the trajectory energy is greater in T3 compared to T1, irrespective of the MC. Accounting for $\mathrm{MC}$ and trial in post hoc contrast analysis, we do not find statistically significant differences. The application of different MCs has no significant effect on the trajectory energy metric in the presented study.

Participants keep minimum distances to the robot that vary between $M=0.37 \mathrm{~m}$ to $M=0.57 \mathrm{~m}$ (see Table 2), which is comparable to accepted distances found in the experiment of Lauckner et al. [32]. In fact, the range is located in between their values for frontal and lateral distances. This could be because in the presented scenario, participants encountered the robot in a lateral formation; however, the subsequent goal direction of human and robot had the same orientation. This formation is different to earlier studies, and thus a comparison of these values may be regarded as approximation at best.

Even though T2 is subsequent to dominant robot behavior, a significant improvement of TFI is found for the $\mathrm{BO}_{\text {short }}$ group. It seems that the $\mathrm{BO}_{\text {short }}$ strategy has an ability to guide humans after variable behavior. The $\mathrm{BO}_{\text {long }}$ strategy does not show the same significant improvement in $\mathrm{T} 2$. The longer TFI in the $\mathrm{BO}_{\text {long }}$ compared to the $\mathrm{BO}_{\text {short }}$ group could occur due to the movement itself being executed with a longer BOD $(0.54 \mathrm{~m}$ compared to $0.19 \mathrm{~m})$ and BOT (3 s compared to $1 \mathrm{~s}$ ). These longer parameters may possibly fall short of their communicative value. For this reason, a distinguished examination of pedestrians that come to a complete standstill inside the IS is performed. To count stopping participants, a walking speed (measured at CLAV) declining to less than $\mathrm{v}<0.4 \mathrm{~m} / \mathrm{s}$ is considered stopping. According to Jian et al. [24], this approximates the speed at which the final brake period of gait termination is initiated and thereby the moment in speed deceleration at which a person touches the ground with the last moving foot before completely decreasing the speed of the body's center of mass to zero. In the ST group $(\mathrm{N}=13)$, three $(23 \%)$, four $(31 \%)$, and five $(39 \%)$ participants stop in $\mathrm{T} 1$ - T3. In the $\mathrm{BO}_{\text {short }}$ group $(\mathrm{N}=15)$, four $(27 \%)$, three $(20 \%)$, and two (13\%) participants stop in $\mathrm{T} 1$ - T3. In the $\mathrm{BO}_{\text {long }}$ group $(\mathrm{N}=18)$, six $(33 \%)$, nine $(50 \%)$, and five $(28 \%)$ participants stop in T1 - T3. Idle times of stopping participants are displayed in Table 2. These mean values indicate that participants who stop their movement take more time to observe the robot's motion in the $\mathrm{ST}$ and the $\mathrm{BO}_{\text {long }}$ group compared to the $\mathrm{BO}_{\text {short }}$ group; however, the number of observations is small. Possibly, the longer backward movement tempts participants to refrain from continuing and excites curiosity about studying the movement, despite having inferred the robot's intent. In $\mathrm{T} 1$ of the $\mathrm{BO}_{\text {long }}$ group, one of these six participants watches the entire BO movement before continuing. This participant continues $3.58 \mathrm{~s}$ after the BO initiation. All other participants continue in less than $1.9 \mathrm{~s}$. In following encounters, all of these observation times are lower than $1.8 \mathrm{~s}$. This may be an indication that a BOT of $3 \mathrm{~s}$ in the $\mathrm{BO}_{\text {long }}$ strategy is more than enough for intent interpretation, and in fact should be decreased to also serve efficiency purposes. Also in the $\mathrm{BO}_{\text {short }}$ group, one of four stopping participants in $\mathrm{T} 1$ watches the entire motion strategy. The participant stands and observes the MC for $1.15 \mathrm{~s}$ after initiation of the robot's backward movement. Other stoppers observe the MC for less than $0.9 \mathrm{~s}$. It seems that the $\mathrm{BO}_{\text {short }}$ provides a sufficiently long backward movement, to communicate intent. Hence, the kinematic parameters BOD and BOT influence human reactions, and may form the 
subject of further studies. Eye-tracking data could give precise insights into duration of fixation. This raises the question of whether there is an optimal minimum BOD and BOT, so humans are able to infer the intent reliably while not distracting the observer, a challenge also found by Pacchierotti et al. [42]. What is more, unnecessarily long BOT causes inefficiency to the robot. Cha et al. [7] demand such optimization, since the cost of a robot's communicative capabilities is often neglected in research. Geometric properties such as the form factor or orientation of the robot could also guide human views. The importance of perspective on the perception of a robot's movement has been emphasized by some authors [38,40]. Only if viewed from the top or the side can the full BOD be perceived. Other viewpoints distort the backward movement. This factor was not varied in the presented experiment. Although it depended on the individual trajectory to approach the bottleneck, the viewpoints were approximately perpendicular to the robot path (see Figure 8). Thus, the study design allowed absolute perception of the BOD.

The additional metrics provide heterogeneous results that are consistent with reports by Mavrogiannis et al. [34]. Navigation strategy has a significant effect on trajectory energy and average acceleration in their study, whereas no significant effect on interaction time is revealed. In the presented experiment, metrics on path smoothness do not agree with the temporal perspective. To conclude, in the presented experiment, time is considered as the indicator for efficiency and the proposition to consider $\mathrm{BO}_{\text {short }}$ for further application relies on the findings regarding the improvement of interaction time and subjective evaluations. Especially, the design of kinematic parameters, $\mathrm{BO}$ path and time, exhibits the potential to increase the fluency of an interaction with robots at bottlenecks in future work.

\section{ACKNOWLEDGMENTS}

We thank Jonas Schmidtler for co-designing the studies; Madeleine Ratter, Sarah Guggenmos, and Sabrina Dinkel for data collection during a student project; and Juliana Berthold and Moritz Körber for proofreading.

\section{REFERENCES}

[1] Klaus Bengler, Markus Zimmermann, Dino Bortot, Martin Kienle, and Daniel Damböck. 2012. Interaction principles for cooperative human-machine systems. it - Information Technology 54, 4 (2012), 157-164.

[2] Andreas Bischof. 2019. Die natürliche Künstlichkeit der Mensch-Roboter-Interaktion als leiblich erfahrbare Irritation des anthropologischen Quadrats. In Mensch und Welt im Zeichen der Digitalisierung. Nomos Verlagsgesellschaft mbH \& Co. KG, Baden-Baden, 127-144.

[3] Richard W. Bohannon. 1997. Comfortable and maximum walking speed of adults aged 20-79 years: Reference values and determinants. Age and Ageing 26, 1 (1997), 15-19.

[4] Daniel Carton, Wiktor Olszowy, and Dirk Wollherr. 2016. Measuring the effectiveness of readability for mobile robot locomotion. International Journal of Social Robotics 8, 5 (2016), 721-741.

[5] Daniel Carton, Annemarie Turnwald, Wiktor Olszowy, Martin Buss, and Dirk Wollherr. 2014. Using penalized spline regression to calculate mean trajectories including confidence intervals of human motion data. In Proceedings of the 2014 IEEE International Workshop on Advanced Robotics and Its Social Impacts. IEEE, Los Alamitos, CA, 76-81.

[6] Elizabeth Cha, Yunkyung Kim, Terrence Fong, and Maja J. Matarić. 2018. A survey of nonverbal signaling methods for non-humanoid robots. Foundations and Trends ${ }^{\circledR}$ in Robotics 6, 4 (2018), 211-323.

[7] Elizabeth Cha, Maja Matarić, and Terrence Fong. 2016. Nonverbal signaling for non-humanoid robots during humanrobot collaboration. In Proceedings of the 11th ACM/IEEE International Conference on Human Robot Interaction. IEEE, Los Alamitos, CA, 601-602.

[8] Herbert H. Clark. 1996. Using Language. Cambridge University Press.

[9] Anca D. Dragan, Kenton C. T. Lee, and Siddhartha S. Srinivasa. 2013. Legibility and predictability of robot motion. In Proceedings of the 8th ACM/IEEE International Conference on Human-Robot Interaction. IEEE, Los Alamitos, CA, 301-308.

[10] M. R. Ensley. 1995. Toward a theory of situation awareness in dynamic systems. Human Factors 37 (1995), $85-104$.

[11] Miguel Faria, Andrea Costigliola, Patrícia Alves-Oliveira, and Ana Paiva. 2016. Follow me: Communicating intentions with a spherical robot. In Proceedings of the 2016 25th IEEE International Symposium on Robot and Human Interactive Communication. IEEE, Los Alamitos, CA, 664-669. https://ieeexplore.ieee.org/document/7745189. 
[12] Claudio Feliciani and Katsuhiro Nishinari. 2015. Phenomenological description of deadlock formation in pedestrian bidirectional flow based on empirical observation. Journal of Statistical Mechanics: Theory and Experiment 2015, 10 (Oct. 2015), P10003.

[13] Anaís Garrell and Alberto Sanfeliu. 2010. Model validation: Robot behavior in people guidance mission using DTM model and estimation of human motion behavior. In Proceedings of the 2010 IEEE/RSF International Conference on Intelligent Robots and Systems. IEEE, Los Alamitos, CA, 5836-5841.

[14] James J. Gibson. 1950. The Perception of the Visual World. Houghton Mifflin, Boston, MA.

[15] Volker Gralla. 2007. Peripheres Sehen im Sport-Möglichkeiten und Grenzen dargestellt am Beispiel der synchronoptischen Wahrnehmung. Ph.D. Dissertation. Ruhr-Universität Bochum.

[16] Herbert Hagendorf, Joseph Krummenacher, Hermann-Joseph Müller, and Torsten Schubert. 2011. Allgemeine Psychologie für Bachelor: Wahrnehmung und Aufmerksamkeit. Springer-Verlag, Berlin, Germany.

[17] Edward T. Hall, Ray L. Birdwhistell, Bernhard Bock, Paul Bohannan, A. Richard Diebold Jr., Marshall Durbin, Munro S. Edmonson, et al. 1968. Proxemics [and comments and replies]. Current Anthropology 9, 2-3 (1968), 83-108.

[18] Ilse Mariska Harms, Joke H. van Dijken, Karel A. Brookhuis, and Dick De Waard. 2019. Walking without awareness. Frontiers in Psychology 10 (2019), 1846.

[19] Fritz Heider and Marianne Simmel. 1944. An experimental study of apparent behavior. American fournal of Psychology 57, 2 (1944), 243-259.

[20] Jean-Michel Hoc. 2001. Towards a cognitive approach to human-machine cooperation in dynamic situations. International fournal of Human-Computer Studies 54, 4 (2001), 509-540.

[21] Guy Hoffman and Cynthia Breazeal. 2007. Effects of anticipatory action on human-robot teamwork efficiency, fluency, and perception of team. In Proceedings of the ACM/IEEE International Conference on Human-Robot Interaction. ACM, New York, NY, 1-8.

[22] Serge P. Hoogendoorn and Winnie Daamen. 2005. Pedestrian behavior at bottlenecks. Transportation Science 39, 2 (2005), 147-159.

[23] Walter Hussy, Margrit Schreier, and Gerald Echterhoff. 2010. Forschungsmethoden in psychologie und sozialwissenschaften-für bachelor. Springer, Berlin, Germany.

[24] Yuancheng Jian, David A. Winter, Milad G. Ishac, and L. Gilchrist. 1993. Trajectory of the body COG and COP during initiation and termination of gait. Gait \& Posture 1, 1 (1993), 9-22.

[25] Wendy Ju. 2015. The design of implicit interactions. Synthesis Lectures on Human-Centered Informatics 8, 2 (2015), $1-93$.

[26] Kay Kitazawa and Taku Fujiyama. 2010. Pedestrian vision and collision avoidance behavior: Investigation of the information process space of pedestrians using an eye tracker. In Pedestrian and Evacuation Dynamics 2008. Springer, Berlin, Germany, 95-108.

[27] Günther Knoblich and Jerome Scott Jordan. 2003. Action coordination in groups and individuals: Learning anticipatory control. Journal of Experimental Psychology: Learning, Memory, and Cognition 29, 5 (2003), 1006.

[28] Sid Kouider, Vincent De Gardelle, Jérôme Sackur, and Emmanuel Dupoux. 2010. How rich is consciousness? The partial awareness hypothesis. Trends in Cognitive Sciences 14, 7 (2010), 301-307.

[29] Thibault Kruse, Alexandra Kirsch, Harmish Khambhaita, and Rachid Alami. 2014. Evaluating directional cost models in navigation. In Proceedings of the 2014 ACM/IEEE International Conference on Human-Robot Interaction. ACM, New York, NY, 350-357.

[30] Chi-Pang Lam, Chen-Tun Chou, Kuo-Hung Chiang, and Li-Chen Fu. 2011. Human-centered robot navigationTowards a harmoniously human-robot coexisting environment. IEEE Transactions on Robotics 27, 1 (2011), 99-112.

[31] Mathis Lauckner. 2016. Human-robot Spatial Interaction in a Hallway. Dissertation. TU Berlin.

[32] M. Lauckner, F. Kobiela, and D. Manzey. 2014. "Hey robot, please step back!" -Exploration of a spatial threshold of comfort for human-mechanoid spatial interaction in a hallway scenario. In Proceedings of the 23rd IEEE International Symposium on Robot and Human Interactive Communication. IEEE, Los Alamitos, CA, 780-787.

[33] Christina Lichtenthäler and Alexandra Kirsch. 2016. Legibility of robot behavior: A literature review. HAL. Retrieved April 1, 2021 from https://hal.archives-ouvertes.fr/hal-01306977.

[34] Christoforos Mavrogiannis, Alena M. Hutchinson, John Macdonald, Patrícia Alves-Oliveira, and Ross A. Knepper. 2019. Effects of distinct robot navigation strategies on human behavior in a crowded environment. In Proceedings of the 2019 14th ACM/IEEE International Conference on Human-Robot Interaction (HRI'19). IEEE, Los Alamitos, CA, 421-430.

[35] Ross Mead and Maja J. Matarić. 2016. Robots have needs too: How and why people adapt their proxemic behavior to improve robot social signal understanding. fournal of Human-Robot Interaction 5, 2 (2016), 48-68.

[36] AJung Moon, Chris A. C. Parker, Elizabeth A. Croft, and H. F. Van der Loos. 2013. Design and impact of hesitation gestures during human-robot resource conflicts. fournal of Human-Robot Interaction 2, 3 (2013), 18-40. 
[37] AJung Moon, Chris A. C. Parker, Elizabeth A. Croft, and H. F. Machiel Van der Loos. 2011. Did you see it hesitate?Empirically grounded design of hesitation trajectories for collaborative robots. In Proceedings of the 2011 IEEE/RSf International Conference on Intelligent Robots and Systems. IEEE, Los Alamitos, CA, 1994-1999.

[38] Yoichi Morales, Takayuki Kanda, and Norihiro Hagita. 2014. Walking together: Side-by-side walking model for an interacting robot. Journal of Human-Robot Interaction 3, 2 (2014), 50-73.

[39] B. C. Muir, S. Rietdyk, and J. M. Haddad. 2014. Gait initiation: The first four steps in adults aged 20-25 years, 65-79 years, and 80-91 years. Gait \& Posture 39, 1 (2014), 490-494.

[40] Stefanos Nikolaidis, Anca Dragan, and Siddharta Srinivasa. 2016. Viewpoint-based legibility optimization. In Proceedings of the 11th ACM/IEEE International Conference on Human-Robot Interaction. IEEE, Los Alamitos, CA, 271-278.

[41] International Federation of Robotics. 2018. Executive Summary World Robotics 2018 Service Robots.

[42] Elena Pacchierotti, Henrik I. Christensen, and Patric Jensfelt. 2006. Evaluation of passing distance for social robots. In ROMAN 2006-The 15th IEEE International Symposium on Robot and Human Interactive Communication. IEEE, Los Alamitos, CA, 315-320.

[43] Jens Rasmussen. 1983. Skills, rules, and knowledge; signals, signs, and symbols, and other distinctions in human performance models. IEEE Transactions on Systems, Man, and Cybernetics SMC-13, 3 (1983), 257-266.

[44] Jakob Reinhardt, Aaron Pereira, Dario Beckert, and Klaus Bengler. 2017. Dominance and movement cues of robot motion: A user study on trust and predictability. In Proceedings of the 2017 IEEE International Conference on Systems, Man, and Cybernetics (SMC'17). IEEE, Los Alamitos, CA, 1493-1498.

[45] Jakob Reinhardt, Jonas Schmidtler, and Klaus Bengler. 2018. Corporate robot motion identity. In Congress of the International Ergonomics Association. Springer, Florence, Italy, 152-164.

[46] Jakob Reinhardt, Jonas Schmidtler, Moritz Körber, and Klaus Bengler. 2016. Follow me! Wie Roboter Menschen führen sollen. Zeitschrift für Arbeitswissenschaft 70, 4 (2016), 203-210.

[47] Jorge Rios-Martinez, Anne Spalanzani, and Christian Laugier. 2015. From proxemics theory to socially-aware navigation: A survey. International fournal of Social Robotics 7, 2 (2015), 137-153.

[48] Matthias Rötting. 2001. Parametersystematik der Augen-und Blickbewegungen für arbeitswissenschaftliche Untersuchungen. Shaker, Düren.

[49] Dorsa Sadigh, Shankar Sastry, Sanjit A. Seshia, and Anca D. Dragan. 2016. Planning for autonomous cars that leverage effects on human actions. In Proceedings of Robotics: Science and Systems, Vol. 2. RSS, Ann Arbor, MI.

[50] Kristian Sandberg, Bert Timmermans, Morten Overgaard, and Axel Cleeremans. 2010. Measuring consciousness: Is one measure better than the other? Consciousness and Cognition 19, 4 (2010), 1069-1078.

[51] Jonas Schmidtler, Christina Harbauer, and Klaus Bengler. 2014. Investigation of human behaviour in pushing and pulling tasks for direct manipulation of a collaborative robot. In Proceedings of the Human Factors and Ergonomics Society Europe Chapter. HFES, Lisbon, Portugal, 15-26.

[52] Jonas Schmidtler, Verena Knott, Christin Hölzel, and Klaus Bengler. 2015. Human centered assistance applications for the working environment of the future. Occupational Ergonomics 12, 3 (2015), 83-95.

[53] Jean Scholtz. 2003. Theory and evaluation of human robot interactions. In Proceedings of the 36th Annual Hawaii International Conference on System Sciences. IEEE, Los Alamitos, CA.

[54] Anna Schubö, Cordula Vesper, Mathey Wiesbeck, and Sonja Stork. 2007. Movement coordination in applied humanhuman and human-robot interaction. In Proceedings of the symposium of the Austrian HCI and Usability Engineering Group. 143-154.

[55] Manfred Schweigert. 2003. Fahrerblickverhalten und Nebenaufgaben. Dissertation. Technical University of Munich.

[56] Natalie Sebanz, Harold Bekkering, and Günther Knoblich. 2006. Joint action: Bodies and minds moving together. Trends in Cognitive Sciences 10, 2 (2006), 70-76.

[57] Natalie Sebanz and Guenther Knoblich. 2009. Prediction in joint action: What, when, and where. Topics in Cognitive Science 1, 2 (2009), 353-367.

[58] Aaron Steinfeld, Terrence Fong, David Kaber, Michael Lewis, Jean Scholtz, Alan Schultz, and Michael Goodrich. 2006. Common metrics for human-robot interaction. In Proceedings of the 1st ACM SIGCHI/SIGART Conference on HumanRobot Interaction. ACM, New York, NY, 33-40.

[59] Razali Tomari, Yoshinori Kobayashi, and Yoshinori Kuno. 2014. Analysis of socially acceptable smart wheelchair navigation based on head cue information. Procedia Computer Science 42 (2014), 198-205.

[60] Thanh Q. Trinh, Christof Schroeter, Jens Kessler, and Horst-Michael Gross. 2015. "Go ahead, please": Recognition and resolution of conflict situations in narrow passages for polite mobile robot navigation. In Proceedings of the International Conference on Social Robotics. 643-653.

[61] Christian Vassallo, Anne-Hélène Olivier, Philippe Souères, Armel Crétual, Olivier Stasse, and Julien Pettré. 2018. How do walkers behave when crossing the way of a mobile robot that replicates human interaction rules? Gait \& Posture 60 (2018), 188-193. 
[62] Heinz Werner. 1935. Studies on contour: I. Qualitative analyses. American fournal of Psychology 47, 1 (1935), $40-64$.

[63] Christopher D. Wickens, Justin G. Hollands, Simon Banbury, and Raja Parasuraman. 2016. Engineering Psychology and Human Performance. Routledge Taylor \& Francis Group, London, UK.

[64] Lars Woyna. 2014. Fahrerzentrierter Kriterienkatalog zur Außensichtbeurteilung im PKW. Dissertation. Technische Universität Darmstadt.

[65] Albert Zaindl. 2018. Wahrnehmungsoptimierte Entwicklung eines Spiegelersatzsystems am Nutzfahrzeug. Dissertation. Technical University of Munich.

Received November 2019; revised May 2020; accepted July 2020 